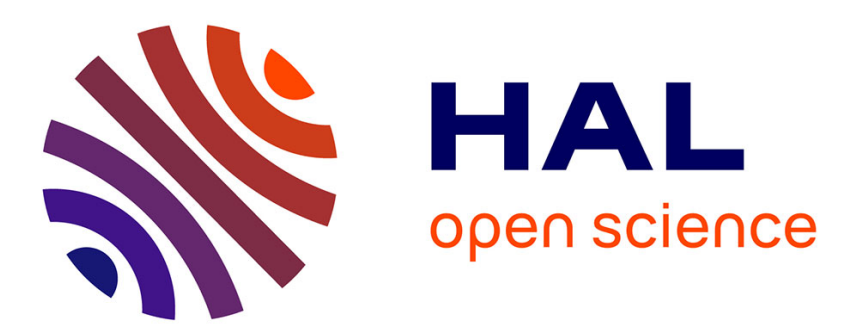

\title{
Control of amplifier flows using subspace identification techniques
}

\author{
Fabien Juillet, Peter J. Schmid, Patrick Huerre
}

\section{To cite this version:}

Fabien Juillet, Peter J. Schmid, Patrick Huerre. Control of amplifier flows using subspace identification techniques. Journal of Fluid Mechanics, 2013, 725, pp.522-565. 10.1017/jfm.2013.194 . hal-00995144

HAL Id: hal-00995144

https://hal-polytechnique.archives-ouvertes.fr/hal-00995144

Submitted on 26 Jun 2014

HAL is a multi-disciplinary open access archive for the deposit and dissemination of scientific research documents, whether they are published or not. The documents may come from teaching and research institutions in France or abroad, or from public or private research centers.
L'archive ouverte pluridisciplinaire HAL, est destinée au dépôt et à la diffusion de documents scientifiques de niveau recherche, publiés ou non, émanant des établissements d'enseignement et de recherche français ou étrangers, des laboratoires publics ou privés. 


\title{
Control of amplifier flows using subspace identification techniques
}

\author{
Fabien Juillet $\dagger$, Peter J. Schmid and Patrick Huerre \\ Laboratoire d'Hydrodynamique (LadHyX), CNRS Ecole Polytechnique, 91128 Palaiseau, France
}

(Received 31 May 2012; revised 31 January 2013; accepted 10 April 2013; first published online 17 May 2013)

A realistic, efficient and robust technique for the control of amplifier flows has been investigated. Since this type of fluid system is extremely sensitive to upstream environmental noise, an accurate model capturing the influence of these perturbations is needed. A subspace identification algorithm is not only a convenient and effective way of constructing this model, it is also realistic in the sense that it is based on input and output data measurements only and does not require other information from the detailed dynamics of the fluid system. This data-based control design has been tested on an amplifier model derived from the Ginzburg-Landau equation, and no significant loss of efficiency has been observed when using the identified instead of the exact model. Even though system identification leads to a realistic control design, other issues such as state estimation, have to be addressed to achieve full control efficiency. In particular, placing a sensor too far downstream is detrimental, since it does not provide an estimate of incoming perturbations. This has been made clear and quantitative by considering the relative estimation error and, more appropriately, the concept of a visibility length, a measure of how far upstream a sensor is able to accurately estimate the flow state. It has been demonstrated that a strongly convective system is characterized by a correspondingly small visibility length. In fact, in the latter case the optimal sensor placement has been found upstream of the actuators, and only this configuration was found to yield an efficient control performance. This upstream sensor placement suggests the use of a feed-forward approach for fluid systems with strong convection. Furthermore, treating upstream sensors as inputs in the identification procedure results in a very efficient and robust control. When validated on the Ginzburg-Landau model this technique is effective, and it is comparable to the optimal upper bound, given by full-state control, when the amplifier behaviour becomes convection-dominated. These concepts and findings have been extended and verified for flow over a backward-facing step at a Reynolds number $R e=350$. Environmental noise has been introduced by three independent, localized sources. A very satisfactory control of the Kelvin-Helmholtz instability has been obtained with a one-order-of-magnitude reduction in the averaged perturbation norm. The above observations have been further confirmed by examining a low-order model problem that mimics a convection-dominated flow but allows the explicit computation of control-relevant measures such as observability. This study casts doubts on the usefulness of the asymptotic notion of observability for convection-dominated flows, since such flows are governed by transient effects. Finally, it is shown that the feedforward approach is equivalent to an optimal linear-quadratic-Gaussian control for spy sensors placed sufficiently far upstream or for sufficiently convective flows. The control design procedure presented in this paper, consisting of data-based subspace

$\dagger$ Email address for correspondence: fabien.juillet@ladhyx.polytechnique.fr 
identification and feed-forward control, was found to be effective and robust. Its implementation in a real physical experiment may confidently be carried out.

Key words: control theory, flow control, instability control

\section{Introduction}

In this paper, different approaches for the control of amplifier flows are presented and compared. A technique consisting of a data-based feed-forward controller is designed and evaluated. It is shown to be at the same time realistic, efficient and robust.

Flow control aims at acting on a fluid system at a few selected locations to induce and enforce a predefined, desired behaviour. Research in this area has received increasing interest fuelled by a large number of potential technological applications in science and engineering. Areas of interest include, among others, drag reduction, control of separation or reattachment, mixing enhancement and the delay of transition to turbulence. Since the way of controlling a fluid critically depends on specific details of the system, a large number of strategies have been designed to manipulate fluid flows. To give a few examples: the actuation may be steady, for instance, constant suction through a flat surface as presented in the review of Joslin (1998), or harmonic, for instance, periodic excitation at a very specific mode frequency as in Greenblatt \& Wygnanski (2000), or it may be based on sensor measurements via a direct feedback, in the simplest case, specified by proportional control.

More generally, a successful control strategy depends equally on the control objective and on the intrinsic flow dynamics. Two flow classes have to be distinguished. Fluid systems known as oscillators are characterized by a periodic behaviour at a sharply defined frequency that is unresponsive to environmental noise sources. Typical examples of this sort of behaviour can be found in hot or swirling jets, mixing layers with sufficiently large counter-flow or flow around a cylinder for a sufficiently high Reynolds number. In contrast, if the flow is strongly influenced by the external disturbance environment, the fluid system is referred to as an amplifier. Pipe or channel flows, co-flowing mixing layers, and boundary layers on a flat plate represent typical examples. If the external noise that drives an amplifier flow is sufficiently small, the fluid system can be described within a linear framework. The classification of fluid flows according to their amplifier or oscillator behaviour was introduced in Huerre \& Monkewitz (1990). The one-dimensional Ginzburg-Landau equation was used as a convenient surrogate for the Navier-Stokes equations. This particular model equation has been popular in addressing a range of related phenomena in fluid mechanics and in flow control, such as in Chomaz, Huerre \& Redekopp (1987) and Lauga \& Bewley (2004).

Within the range of active control strategies, a model-based approach has been prevalent in the flow control literature (see e.g. Kim \& Bewley 2007; Noack, Morzynski \& Tadmor 2011). Its underlying premise is to determine a mathematical model that accurately describes the system dynamics and to subsequently use it to design an optimal control law. Several strategies are available to arrive at such a model. For sufficiently simple systems, a model equation can be deduced directly from physical principles (Bewley \& Liu 1998). For more complex problems, numerical simulations offer an alternative to obtain this model (Semeraro et al. 2011). Both 
approaches provide valuable insight into the physical mechanisms of instability and flow control. However, both approaches also suffer from the need to accurately describe the external disturbance environment. In many previous studies (Lauga \& Bewley 2004; Bagheri, Brandt \& Henningson 2009a; Chen \& Rowley 2011; Ma, Ahuja \& Rowley 2011; Semeraro et al. 2011; Barbagallo et al. 2012), a simplified model for the disturbance environment is assumed in the control design. The resulting control performance is somewhat contrived, since it is closely tailored to the postulated noise model. A similar approach is inconceivable in an experimental setting. Another approach resorts to system identification theory in order to generate a model. In particular, this technique has been successfully applied experimentally with several goals such as the suppression of flow-induced cavity tones (Cattafesta et al. 2003; Kegerise, Cabell \& Cattafesta 2004), the reattachment of a separated boundary layer over a pitched aerofoil (Tian, Song \& Cattafesta 2006), the manipulation of the reattachment point downstream of a backward-facing step (Henning \& King 2007) or the control of lift of an aerofoil in the presence of gusts (Kerstens et al. 2011). A rather recent approach consists of using system identification techniques to obtain a model that directly approximates the linearized Navier-Stokes equations. In this context, promising results have been found by Hervé et al. (2012). The principal advantage of this approach lies in the fact that the model is directly derived from experimental, and thus noise-contaminated, data. In this sense, such a technique may provide a better description of a realistic (experimental) fluid system. However, a physical interpretation of the identified model may prove to be rather challenging.

System identification is concerned with building input-output models for dynamical systems directly from input-output observations. Even though this field of research comprises a wide range of techniques and applications, we restrict our attention to algorithms developed for the identification of stable linear time-invariant (LTI) systems. Applying these techniques to fluid systems may be justified in the case of amplifier flows if they are excited by small perturbations. In that case, the flow dynamics can be decomposed as a sum of a steady base flow (time-invariant) and a perturbation flow field. For sufficiently small perturbations, the amplifier behaviour is then accurately described by the Navier-Stokes equations linearized around the base flow, and system identification techniques therefore aim at constructing a model that accurately captures any input-output behaviour of the linearized Navier-Stokes operator.

If the underlying model is specified - for instance, as an autoregressive model with exogenous inputs (ARX), or as an autoregressive moving-average model with exogenous inputs (ARMAX) - the identification technique proceeds by matching the input-output behaviour of the model to the observed data in a least-squares sense (Ljung 1999). An alternative is to identify the discrete linear system matrices by computing estimates of the state vector over many consecutive time steps. This technique is referred to as subspace identification; the main idea stems from Kalman (1960) and the method has been formalized by Larimore (1983). Commonly used subspace identification algorithms include the canonical variate analysis (CVA) algorithm (Larimore 1983, 1990), the multiple-inputs and multiple-outputs output-error state-space algorithm MOESP (Verhaegen \& Deprettere 1991) and the numerical algorithms for subspace state-space system identification N4SID (Van Overschee \& De Moor 1994). A comprehensive description of these techniques, within a unified framework, is given in Van Overschee \& De Moor (1996); for a more recent review on the subject, see for instance Qin (2006). In addition to the linear system matrices, subspace identification techniques provide an approximation of the noise covariances, which are required for a subsequent control design based on linear-quadratic-Gaussian 
(LQG) theory. In fact, subspace identification and LQG control design are two intimately related procedures, and the corresponding approaches can be combined into a single technique that produces an optimal control strategy directly from the input-output data sequences (Favoreel et al. 1998).

Over the last two decades, the LQG framework has provided the central component of many flow control studies, as in Bewley \& Liu (1998), Bewley (2001), Högberg \& Henningson (2002), Chevalier et al. (2007), Bagheri et al. (2009a) and Semeraro et al. (2011). The appeal - and thus widespread use - of this technique lies in its theoretical optimality. A great many other alternatives are, however, available, and even preferred in robust control applications for industrial problems; this over-emphasis on LQG control has also been noted by Qin \& Badgwell (2003).

This paper consists in a presentation and evaluation of a combined approach involving subspace identification and optimal control design. In particular, we are interested in a design that is not only efficient in reducing noise amplification, but also robust in its performance and realistic with respect to available system information. The successful application of flow-altering momentum or energy sources necessarily relies on efficient strategies and control laws. These control laws, in turn, have to be insensitive to small variations in the underlying assumptions, to model approximations or to parameter uncertainties. Only schemes that exhibit this property, i.e. robust schemes, will be able to perform under a range of environmental conditions, rather than at a narrowly defined or idealized design point. Effective control strategies also have to be realistic in a sense that their design only relies on quantities that are readily available in numerical simulations as well as in physical experiments; this precludes the use of purely mathematical quantities such as system matrices or prescribed stochastic disturbance environments. In this paper we propose and analyse a feed-forward design procedure that accomplishes these three objectives. Feed-forward control is not a new approach and it has been used intensively in other fields of research such as in automatics (see e.g. Meckl \& Seering 1986), in chemistry (see e.g. Calvet \& Arkun 1988) or in noise cancellation application (see e.g. Zeng \& de Callafon 2003) or temperature control (see e.g. Thomas, Soleimani-Mohseni \& Fahln 2005). A recent and successful numerical application of such a strategy for the control of flow over a backward-facing step can be found in Hervé et al. (2012). The main purpose of the present paper is to identify and understand the strengths and weaknesses of the feed-forward approach when applied to amplifier flows. In particular, it will be seen that convection plays a crucial role in the relative efficiency of the technique.

The paper is organized as follows. In $\S 2$, subspace identification techniques are introduced and the LQG framework is briefly discussed. Based on these techniques, a standard control approach is applied to the linear Ginzburg-Landau equation in $\S 3$ and its limitations are pointed out. In particular, the relative estimation error and the introduction of the concept of a visibility length are shown to provide valuable tools in analysing the strengths and weaknesses of standard feedback control in convection-dominated flows $(\$ 3.4)$. In this respect, it is advantageous to place sensors further upstream in the flow domain. The resulting feed-forward approach leads to the design of a realistic, robust and efficient control scheme $(\S 4)$. The technique is then validated on a more realistic fluid system, namely flow over a backward-facing step at a Reynolds number $R e=350(\S 5)$. Finally, this successful feed-forward strategy is further explored and analysed in mathematical terms and it is compared to the optimal LQG control design ( $\S 6$ ). A discussion of the main conclusions is given in $\S 7$. For the 
sake of clarity, the details of the presentation of the subspace identification algorithm have been relegated to the appendix A.

\section{System definition and control performance}

\subsection{System definition}

The one-dimensional linear Ginzburg-Landau equation (see e.g. Bagheri et al. 2009b) is selected as a model problem. It reads

$$
\frac{\partial q}{\partial t}=-\left(U_{r}+\mathrm{i} U_{i}\right) \frac{\partial q}{\partial x}+\left(1+\mathrm{i} c_{d}\right) \frac{\partial^{2} q}{\partial x^{2}}+\mu(x) q,
$$

where $q(x, t)$ denotes the state, which is assumed bounded as $|x|$ tends to infinity. This equation is widely used as a model in fluid mechanics due to its convectively or absolutely unstable characteristics (Huerre \& Monkewitz 1990; Lauga \& Bewley 2004). It contains convective and diffusive/dispersive terms, as well as a local instability governed by the parameter $\mu(x)$. In the present case, the latter function is chosen as $\mu(x)=\mu_{1}-\left(U_{i} / 2\right)^{2}+\mu_{2} x^{2} / 2$, with $\mu_{1}=0.38, \mu_{2}=-0.01$ and $U_{i}=0.4$, such that only a finite region, given by $|x|<\sqrt{2 \mu_{1} /\left|\mu_{2}\right|} \approx 8.72$, is locally unstable. The parameters of the Ginzburg-Landau equation are the same as in Bagheri et al. (2009b) and Chen \& Rowley (2011); only the convection coefficient $U_{r}$ may differ when specified. More precisely, the dispersion coefficient $c_{d}$ is equal to -1 and two different values of the convection speed $U_{r}$ are selected. To model a fluid system in the presence of moderate convection, the value $U_{r}=2$ is chosen, whereas $U_{r}=3$ corresponds to a case of large convection. With this choice of parameters, the flow is always globally stable, even though a sizable absolutely unstable region is present in the case of moderate convection $U_{r}=2$, a feature that can be attributed to the non-parallel nature of the flow stemming from the $\mu(x)$ term (Chomaz et al. 1987; Huerre \& Monkewitz 1990; Bagheri et al. 2009b). Systems of this type are referred to as amplifiers, i.e. any perturbation moving into the unstable region is convected, filtered and amplified.

It is further assumed that the governing equation is excited by a noise source that is localized inside the upstream locally stable domain, at $x_{w}=-14$. The response to an impulse from this noise location gives insight into the system dynamics. It is displayed in figure 1(a) with isocontours of $|q|$ in an $x-t$ diagram. For moderate convection speed $U_{r}=2$, the impulse decays initially (in the stable region) but grows rather rapidly as it moves into the convectively unstable region. More quantitatively, the maximum value of $q$ is less than 0.01 as the perturbation enters the unstable region (at $t \approx 2$ ), but it is amplified by a factor of more than four at $t=20$ before it slowly decays for $t>20$. This is in contrast to the impulse response for a large convection speed $U_{r}=3$ displayed in figure $1(b)$. In this case, a similar, initially convected pattern is observed, but the slowly decaying quasi-stationary perturbation is conspicuously absent. This strong difference in the behaviour of the two systems $\left(U_{r}=2\right.$ and $U_{r}=3$ ) is attributed to the presence of an absolutely unstable region in the moderate convection case $\left(U_{r}=2\right)$.

Hence, within the family of amplifier flows, very different behaviours are observed, and it will be seen in the next section that this has a direct impact on the efficiency of any control strategy. More precisely, two cases are distinguished, one with moderate convection and one with large convection. In fact, convection is a central component of amplifier flows. From a local point of view, a parallel flow is an amplifier if it is convectively unstable, that is, if, for infinitely large times, any unstable wave is 

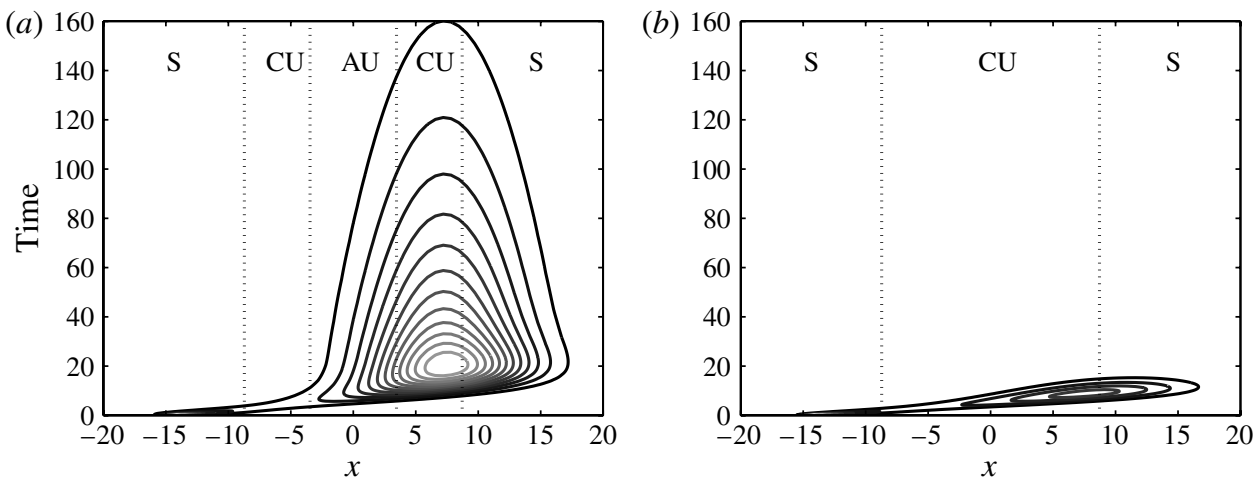

FIGURE 1. Response to an impulse from the noise location shown as $|q|$ contours in an $x-t$ diagram: $(a)$ for a moderate convection speed $U_{r}=2$ and $(b)$ for a large convection speed $U_{r}=3$. The absolutely unstable (AU), convectively unstable (CU) or stable (S) spatial domains are marked.

associated with a non-zero group velocity (Huerre \& Monkewitz 1990). From a global point of view, convection is responsible for the non-normality of the linear operator in globally stable open flows, which, consequently, results in an amplifier behaviour (Chomaz 2005). Hence, it is clear that amplifier flows are necessarily associated with a non-negligible convection. The moderate convection $U_{r}=2$ corresponds to a case where convection is sufficiently high for the flow to be globally stable and to be classified as an amplifier. Yet this convection is relatively small and, as illustrated in figure 1(a), the competition between local instabilities and convection has a very strong impact on the intrinsic global flow behaviour.

Thus, within the set of amplifier flows, a flow is convection-dominated if its behaviour is mainly governed by convective processes. For instance, pipe or channel flows, homogeneous jet flows, or flows over streamlined aerofoils at small angles of attack are examples of convection-dominated flows. In the present study, as seen in figure 1, the large convection case $U_{r}=3$ has a convection-dominated behaviour whereas the moderate convection case $U_{r}=2$ does not. It will be seen in $\$ 4.2$ that the technique developed in this paper is particularly efficient for convection-dominated flows.

To complete the specification of the control set-up, a sensor and an actuator are placed at $x_{y}=7$ and $x_{u}=0$, respectively, with a Gaussian shape function of width $s=0.4$ (figure 2). In fact, the sensor placement corresponds to the position where the impulse response is largest (figure 1). Hence this sensor provides direct information on the maximum amplification within the flow.

Following Bagheri et al. (2009b), the Ginzburg-Landau equation is discretized in space using a pseudo-spectral method based on Hermite functions where $n=220$ nodes are distributed within the interval $-85<x<85$. The discrete state vector is advanced in time by a Crank-Nicolson scheme with a constant time step $\Delta t=0.1$. The spatio-temporal discretization then yields the discrete state-space formulation

$$
\begin{aligned}
q_{k+1} & =\boldsymbol{A} q_{k}+\boldsymbol{B} u_{k}+\boldsymbol{B}_{w} w_{k}, \\
y_{k} & =\boldsymbol{C} q_{k}+v_{k},
\end{aligned}
$$

where $q_{k}$ is a column vector with $n$ components describing the state at time $t=k \Delta t, \boldsymbol{A}$ is the state matrix of size $n \times n, \boldsymbol{B}$ is the actuator input matrix of size $n \times 1, \boldsymbol{B}_{w}$ is the 
(a)

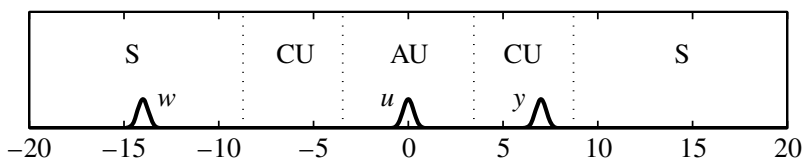

(b)

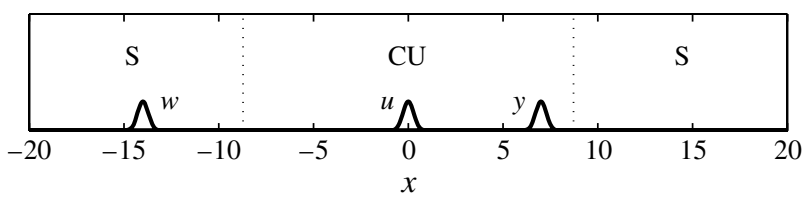

FIGURE 2. Sketch of the control set-up for the linear Ginzburg-Landau equation, showing the noise source $w$ at $x_{w}=-14$, the actuator $u$ at $x_{u}=0$ and the sensor $y$ at $x_{y}=7$ for (a) moderate convection $U_{r}=2$ and $(b)$ large convection $U_{r}=3$.

noise input matrix of size $n \times 1$ and $\boldsymbol{C}$ is the output matrix of size $1 \times n$. In addition, $w_{k}$ is a stochastic, normally distributed, white-in-time noise of standard deviation $\sigma_{W}=1$, $u_{k}$ is the actuator input, $y_{k}$ is the sensor output and $v_{k}$ is the measurement noise (again, normally distributed and white-in-time of standard deviation $\sigma_{V}$ equal to $10 \%$ of the output standard deviation when the system is excited by $w$ only). From the stochastic terms of the above system, noise covariances $\boldsymbol{R}, \boldsymbol{S}$ and $\boldsymbol{Q}$ can be defined according to

$$
\mathscr{E}\left(\left(\begin{array}{c}
\boldsymbol{B}_{w} w_{k} \\
v_{k}
\end{array}\right)\left(\begin{array}{c}
\boldsymbol{B}_{w} w_{p} \\
v_{p}
\end{array}\right)^{H}\right)=\left(\begin{array}{cc}
\boldsymbol{Q} & \boldsymbol{S} \\
\boldsymbol{S}^{H} & \boldsymbol{R}
\end{array}\right) \delta_{p k},
$$

where $\mathscr{E}(a) \equiv \lim _{N \rightarrow \infty}(1 / N) \sum_{k=1}^{N} a_{k}$ denotes the expected value and the superscript ${ }^{H}$ symbolizes the conjugate transpose. In practice, $v$ and $w$ are uncorrelated such that $\boldsymbol{S}$ is identically zero. Recall that $\sigma_{W} \equiv 1$ and $\sigma_{V} \equiv \sqrt{\boldsymbol{R}}$.

\subsection{Control performance}

We next design a control scheme based on the set-up above and determine its effectiveness in reducing the upstream noise $w$. Ideally, the norm of the state vector, $\|q\|$, is to be minimized. In practice, however, the state vector cannot be measured directly; instead, only information from the sensor is available. Since the measurement is performed at $x_{y}=7$, where the impulse response amplitude is largest (figure 1a), it is reasonable to assume that by reducing the output $y$ a commensurate reduction of the state vector $q$ can be accomplished. We thus formulate the cost functional $J$ as

$$
J \equiv \mathscr{E}\left(\|y\|^{2}+l\|u\|^{2}\right) \rightarrow \min ,
$$

where $l$ is a positive parameter that penalizes the exerted control effort and thus prevents excessive input amplitudes, and $\|\cdot\|$ represents the Euclidean norm. In what follows we set $l=0.001$. The state-space system (2.2) and the cost functional (2.4) are augmented by an optimal control law (LQG) based on the measurement $y$. The results are presented in figure $3(a)$ for a moderate convection speed $U_{r}=2$. The full-order system $(\boldsymbol{A}, \boldsymbol{B}, \boldsymbol{C})$ as well as the noise covariances $(\boldsymbol{Q}, \boldsymbol{R}, \boldsymbol{S})$ are assumed to be given. The quantity $J_{k}=\left\|y_{k}\right\|^{2}+l\left\|u_{k}\right\|^{2}$ is represented as a function of time, together with its expected values for an active and inactive control $u$. At $t=400$ the control is switched on, and the cost functional rapidly decreases by nearly two orders of magnitude, settling down to an expected value $J=0.0064$ from an uncontrolled value $J=0.25$, i.e. a reduction by a factor of 38 . 


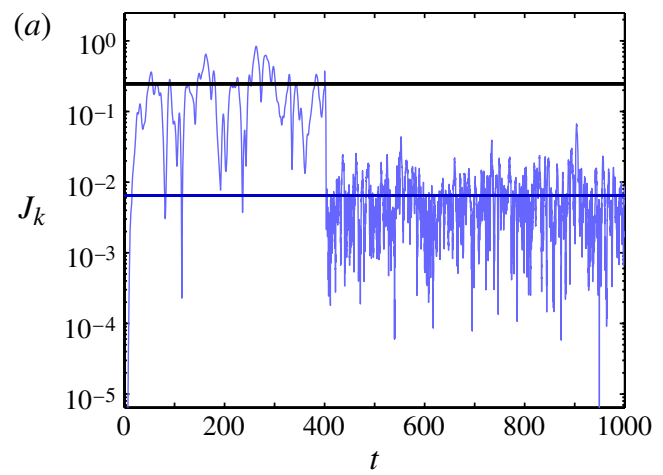

(b)

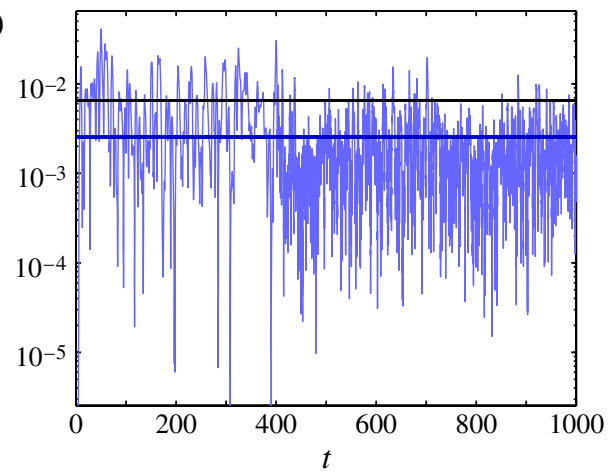

FIGURE 3. (Colour online) Cost functional $J=\mathscr{E}\left(y^{2}+l u^{2}\right)$ with control (in grey; blue online) and without control (in black), for $(a)$ moderate convection $U_{r}=2$ and $(b)$ large convection $U_{r}=3$. In addition the quantity $J_{k}=y_{k}^{2}+l u_{k}^{2}$ is plotted as a function of time. The control is turned on at $t=400$. The LQG controller has been designed based on the full-order system (2.2) and the full-order noise covariances (2.3).

The same control design procedure has also been applied to the case of the large convection speed $U_{r}=3$. The control performance measure is shown in figure $3(b)$. The optimal control in seen to achieve a reduction in the cost functional of only a factor 2.6. This decreased performance results from two considerations. First, for $U_{r}=2$ the perturbation remains in the unstable region for more than 1500 time steps (figure $1 a$ ), and the control can be applied over this entire time span, while for $U_{r}=3$ the perturbation decays substantially more rapidly (figure $1 b$ ), and the control has a very limited 'window of opportunity' (when the perturbation passes the actuator location) to accomplish its objective. The second observation is related to the efficiency of the estimation process. For a large convection speed (in our case $U_{r}=3$ ), the information from the sensor downstream fails to sufficiently capture the upstream dynamics of the incoming perturbation, which is essential for effective control.

In the previous computation, the LQG framework was chosen to probe the optimal control performance for a given actuator-sensor set-up and to quantify the control efficiency that may be expected, in the best case. The disappointing reduction in performance for convection-dominated flows $\left(U_{r}=3\right)$ cannot be attributed to the control technique, but rather it has to be related to the control set-up. This first issue is dealt with in $\S 3.4$. Furthermore, it has to be kept in mind that, while establishing an upper bound for control performance, the LQG design involves mathematical quantities such as $\boldsymbol{A}, \boldsymbol{B}, \boldsymbol{C}, \boldsymbol{Q}, \boldsymbol{R}$ and $\boldsymbol{S}$ that are generally not available in physical experiments. To address this issue, a technique that only depends on realistically available quantities is introduced in the next section. It extracts the system matrices and noise covariances directly from measured data sequences. The efficiency of this scheme will then be compared with the optimal (but unrealistic) LQG standard.

\section{Towards a realistic LQG control scheme based on subspace identification}

\subsection{System identification by subspace techniques}

System identification is a well-established technique for the recovery of deterministic and/or stochastic dynamical systems from their response to input signals. It comprises 
Step 1:

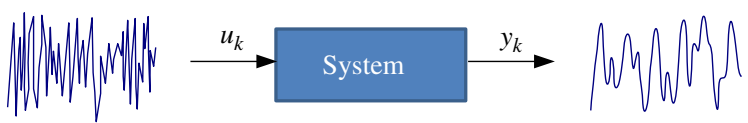

Step 2:

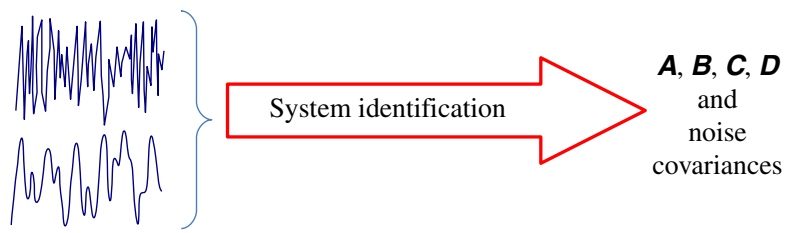

Step 3:

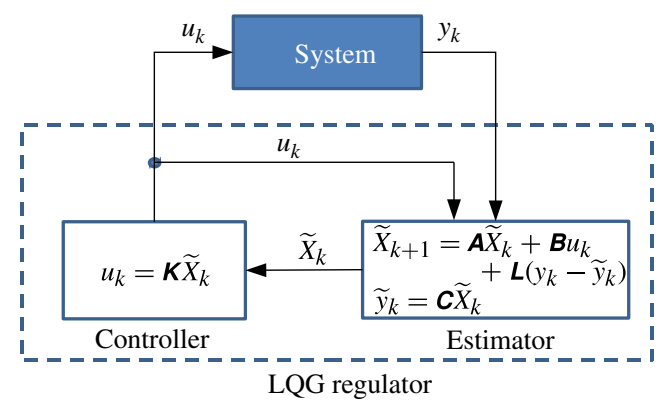

FIgURE 4. (Colour online) Procedural steps of control design based on system identification. Step 1: excitation of the system with a known input signal and simultaneous measurement of the output. Step 2: subspace identification of the model. Step 3: design of the LQG regulator and compensation of the system.

numerous methods of varying applicability and complexity (Ljung 1999). In the present case, we are interested in extracting the system matrices $(\boldsymbol{A}, \boldsymbol{B}, \boldsymbol{C})$ together with the noise covariances $(\boldsymbol{Q}, \boldsymbol{R}, \boldsymbol{S})$ by processing measured data sequences for $u$ and $y$. The identification of noise covariances is particularly important for flow amplifiers where the response to noise plays a crucial role. For this reason, a subspace identification technique, which provides approximations of the noise covariances $(\boldsymbol{Q}, \boldsymbol{R}, \boldsymbol{S})$, is chosen. A comprehensive description of these techniques can be found in Van Overschee \& De Moor (1996) or Qin (2006). The procedural steps of subspace identification and control design are sketched in figure 4. First, the system is excited by an arbitrary, but frequency-rich input signal $u$ and by unknown disturbances $w$; meanwhile, the output signal $y$ is recorded. In a second step, the known input and output signals are processed and a subspace identification algorithm provides a linear model $(\boldsymbol{A}, \boldsymbol{B}, \boldsymbol{C})$ together with the noise covariances $(\boldsymbol{Q}, \boldsymbol{R}, \boldsymbol{S})$. In a final step, an LQG regulator is designed based on this model. The subspace identification algorithm constitutes a central element in the entire analysis. The reader is referred to appendix A for its detailed presentation. The paper may be read independently of appendix A.

\subsection{Linear-quadratic-Gaussian (LQG) framework}

Once input-output data sequences have been used to identify the system matrices and noise covariances, we are in a position to design an estimator and controller using the LQG framework and to compensate the identified system (step 3 in figure 4). This design step is well covered in the flow control literature (Friedland 1986; Skogestad \& 
Postlethwaite 1996; Zhou, Doyle \& Glover 1996; de Larminat 2002) and thus it will be only briefly reviewed for completeness.

The objective of the control is to find a sequence $u_{k}$ such that the cost functional $J$ given by

$$
J=\mathscr{E}\left(\|y\|^{2}+l\|u\|^{2}\right)
$$

is minimized, where $y$ is governed by the state-space system

$$
\begin{aligned}
q_{k+1} & =\boldsymbol{A} q_{k}+\boldsymbol{B} u_{k}+\boldsymbol{B}_{w} w_{k}, \\
y_{k} & =\boldsymbol{C} q_{k}+v_{k},
\end{aligned}
$$

with given system matrices $\boldsymbol{A}, \boldsymbol{B}, \boldsymbol{C}$, known noise covariances and measured output $y_{k}$. In a first step, the full-state control problem is solved, which assumes that the state $q_{k}$ is entirely known. With a linear optimal control law, i.e. $\boldsymbol{u}_{k}=\boldsymbol{K} q_{k}$, the full-state control problem can be expressed in the form of an optimization problem: find a constant gain matrix $\boldsymbol{K}$ such that the cost functional

$$
J=\mathscr{E}\left(q^{H}\left(\boldsymbol{C}^{H} \boldsymbol{C}+l \boldsymbol{K}^{H} \boldsymbol{K}\right) q\right)
$$

is minimized, where $q$ solves $(3.2 a)$. If $(\boldsymbol{A}, \boldsymbol{B})$ is stabilizable, i.e. there exists a matrix $\boldsymbol{M}$ such that $\boldsymbol{A}-\boldsymbol{B} \boldsymbol{M}$ is stable, and $(\boldsymbol{C}, \boldsymbol{A})$ is detectable, i.e. there exists a matrix $\boldsymbol{M}$ such that $\boldsymbol{A}-\boldsymbol{M C}$ is stable, a symmetric, positive semi-definite matrix $\boldsymbol{T}$ can be uniquely determined (Zhou et al. 1996; de Larminat 2002) as a solution of the discrete algebraic Riccati equation:

$$
\boldsymbol{T}=\boldsymbol{A}^{H} \boldsymbol{T A}-\boldsymbol{A}^{H} \boldsymbol{T} \boldsymbol{B}\left(\boldsymbol{I}+\boldsymbol{B}^{H} \boldsymbol{T} \boldsymbol{B}\right)^{-1} \boldsymbol{B}^{H} \boldsymbol{T A}+\boldsymbol{C}^{H} \boldsymbol{C} .
$$

The optimal control gain $\boldsymbol{K}$ is then given by

$$
\boldsymbol{K}=-\left(l+\boldsymbol{B}^{H} \boldsymbol{T} \boldsymbol{B}\right)^{-1} \boldsymbol{B}^{H} \boldsymbol{T A},
$$

and the controlled system is stable.

In practice, the state $q$ is not available and an estimator has to be designed that approximately, but optimally, recovers the state from measurements $y_{k}$ only. The estimation problem reads

$$
\begin{aligned}
\tilde{q}_{k+1} & =\boldsymbol{A} \tilde{q}_{k}+\boldsymbol{B} u_{k}+\boldsymbol{L}\left(y_{k}-\tilde{y}_{k}\right), \\
\tilde{y}_{k} & =\boldsymbol{C} \tilde{q}_{k},
\end{aligned}
$$

where the Kalman gain $\boldsymbol{L}$ has to be optimized so that the estimation error, i.e. the difference between the true state $q_{k}$ and the estimated state $\tilde{q}_{k}$, is minimized. Under the assumption that $(\boldsymbol{C}, \boldsymbol{A})$ is detectable and $\left(\boldsymbol{A}, \boldsymbol{B}_{w}\right)$ is stabilizable, this optimization problem again leads to a discrete algebraic Riccati equation for a unique, symmetric, positive definite matrix $\boldsymbol{P}$ according to

$$
\boldsymbol{P}=\boldsymbol{A P A ^ { H }}+\boldsymbol{Q}-\boldsymbol{A P C ^ { H }}\left(\boldsymbol{R}+\boldsymbol{C P} \boldsymbol{C}^{H}\right)^{-1} \boldsymbol{C P \boldsymbol { A } ^ { H }} .
$$

The optimal Kalman gain $\boldsymbol{L}$ then follows as

$$
\boldsymbol{L}=\boldsymbol{A P C ^ { H }}\left(\boldsymbol{Q}+\boldsymbol{C P} \boldsymbol{C}^{H}\right)^{-1}
$$

Combining the controller and estimator yields a compensator, which produces an optimal control strategy $u_{k}$ directly from measurements $y_{k}$. Even though the control 

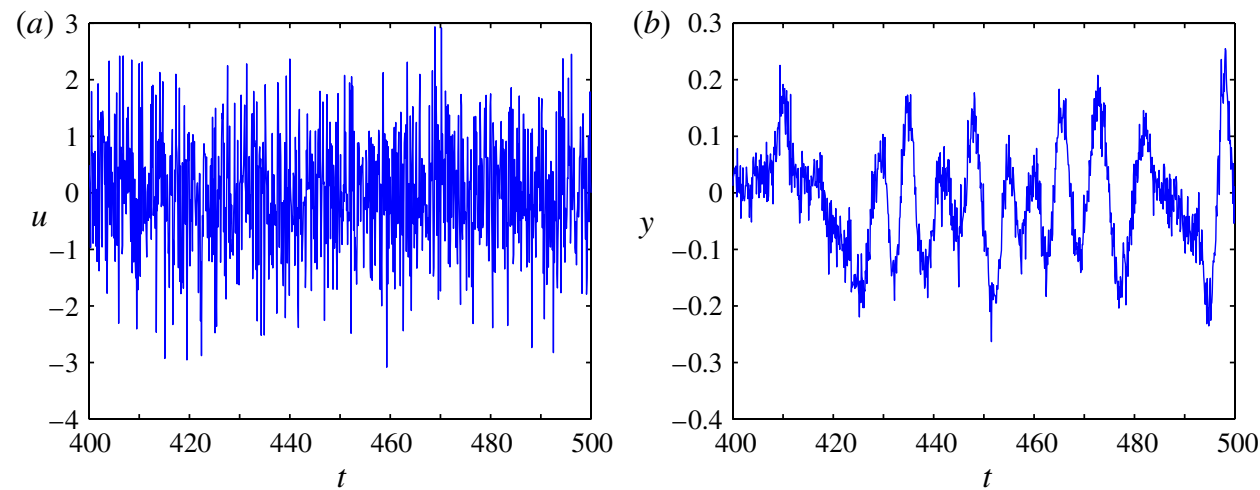

FIgURE 5. (Colour online) Sample of the $(a)$ input and $(b)$ output signal used for the subspace identification for the large convection case $U_{r}=3$. The full signal is over 500 time units, i.e. 5000 time steps long. The input signal $(a)$ is chosen to be Gaussian and white; the measured signal $(b)$ is coloured by the system.

gain $\boldsymbol{K}$ has been designed under full-state assumptions, it still remains optimal when used with the estimated (rather than the exact) state. This fact is a consequence of the separation theorem (Friedland 1986; Skogestad \& Postlethwaite 1996), which states that the design of the optimal controller and the optimal estimator can be performed independently.

\subsection{A first attempt at $L Q G$ control design}

The identification technique, introduced in $\S 3.1$ and presented in appendix A, produces all the necessary system and noise information from measured data. It ensures the applicability of the overall control design to experimental data. In this respect, the subspace identification technique guarantees the realistic aspect of the control design. In the following, the efficiency and robustness of the approach are evaluated.

From the identified system matrices $\boldsymbol{A}, \boldsymbol{B}, \boldsymbol{C}$ and noise covariances $\boldsymbol{R}, \boldsymbol{S}, \boldsymbol{Q}$, we follow the classical control design within the LQG framework, the third and final step in figure 4 . We choose a large convection speed $U_{r}=3$, starting with the sensor-actuator configuration shown in figure 2. A set of input-output samples consisting of 5000 discrete values is generated from a Gaussian, white input signal. A representative sample of input and output signals is shown in figure 5. We observe that, as the Gaussian, white input signal passes through the linear system, it retains some frequencies while attenuating others, thereby resulting in a coloured signal for the output. From these signals a reduced-order model of order four is identified using the subspace identification algorithm MOESP (see $\S 3.1$ and appendix A).

The impulse response of the identified model is compared to the impulse response of the true system in figure 6 . We note an overall satisfactory match, despite small discrepancies for larger times, which can be attributed to the appreciable level of environmental noise in the output signal. It is still remarkable for a model of order four to reproduce the response behaviour of a system of order $n=220$.

The optimal control gain $\boldsymbol{K}$ is then obtained, for the large convection case $U_{r}=3$, by solving the appropriate discrete algebraic Riccati equation (3.4) using the identified system matrices. The optimal estimation (Kalman) gain $\boldsymbol{L}$ is determined by using the 


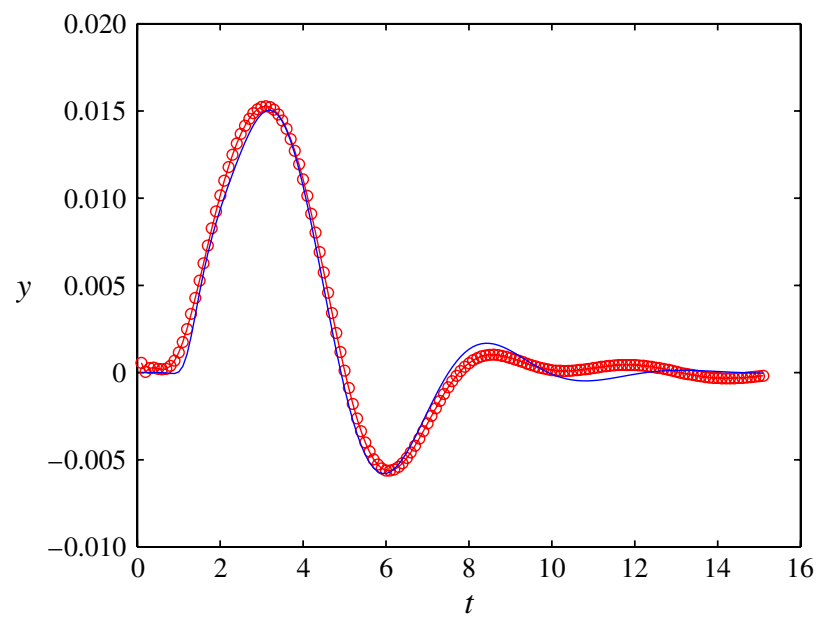

FIGURE 6. (Colour online) Impulse response (from $u$ to $y$ ) of the full-order system (thin line; blue online) compared to the impulse response of the identified system (circles; red online).

identified system matrices and noise covariances in a second discrete algebraic Riccati equation (3.7). In figure 7 the quantity $J_{k}=\left\|y_{k}\right\|^{2}+l\left\|u_{k}\right\|^{2}$ is presented as a function of time, and the control is turned on after 4000 time steps. Three cases are considered. The most effective, but idealistic, control strategy (in blue) is based on full-state control, as it assumes knowledge of the full system matrices $\boldsymbol{A}, \boldsymbol{B}, \boldsymbol{C}$ and even access to the state vector $q$. The second case (in green) reproduces the results displayed in figure 3. This still unrealistic control strategy relies on the system matrices $\boldsymbol{A}, \boldsymbol{B}, \boldsymbol{C}$ but substitutes an estimated state $\tilde{q}$ for the true state $q$. The only realistic, third, case assumes no prior knowledge of the system matrices, but extracts all the necessary information from measured data. The identified matrices and noise covariances are then used to design a compensator. The terms full-state control, full-order control and identified-model-based control will be used throughout the paper to designate these three distinct control approaches.

From figure 7 we conclude that the loss of control performance cannot be attributed to the identification step, as it does not appear to significantly degrade the efficiency of the control strategy. For this reason, we can safely rely on subspace identification as a crucial step towards realistic control applications. Rather, the comparison of the three cases points towards the estimation process as the component that deteriorates the control performance. This is reflected in the marked gap between the cost reduction, by a factor of 166, for the estimation-free (full-state) control and the disappointing low cost reduction, by a factor of 2.6, for the estimation-based (full-order) control.

\subsection{Visibility and optimal placement of a 'spy' sensor}

A closer look at the estimation process is now taken by computing the standard deviation of the estimation error, in the statistically stationary limit, for each position in $x$. More precisely, the standard deviation of the estimation error is defined as $\operatorname{std}(e) \equiv \sqrt{\operatorname{diag}\left(\mathscr{E}\left((\tilde{q}-q) \cdot(\tilde{q}-q)^{H}\right)\right)}$, where $\operatorname{diag}(A)$ is the vector constructed with the diagonal elements of any square matrix $\boldsymbol{A}$, the state $q$ and the estimated state $\tilde{q}$ are defined in (3.2) and (3.6), respectively, and $\mathscr{E}$ is the expected value as defined in (2.3). Similarly the standard deviation of the state is defined as $\operatorname{std}(q) \equiv \sqrt{\operatorname{diag}\left(\mathscr{E}\left(q \cdot q^{H}\right)\right)}$. 


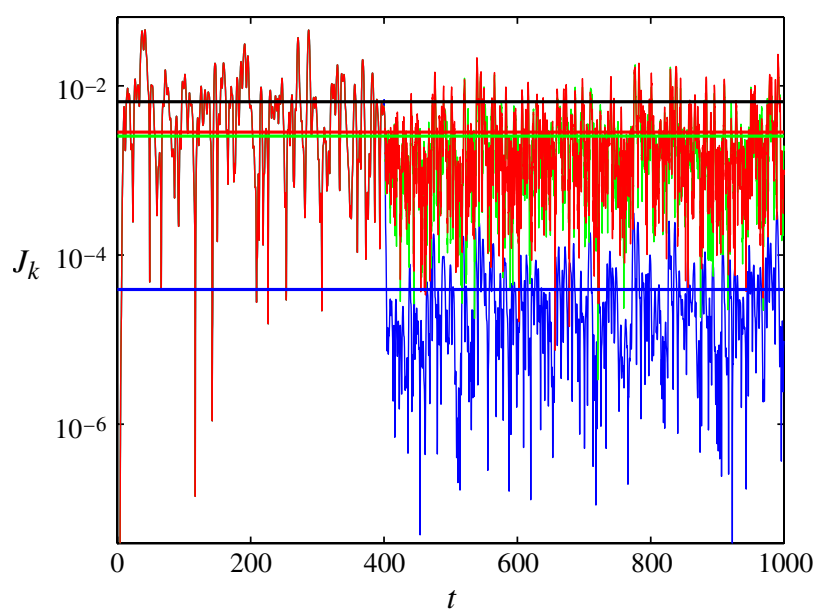

FIgURE 7. Cost functional $J=\mathscr{E}\left(y^{2}+l u^{2}\right)$ as a function of time without control (in black) and with control applied (in green, red and blue). The blue line represents full-state control designed from knowledge of all full-order system matrices as well as $q$. The green line represents LQG control designed from the full-order system matrices and noise covariances (same as in figure $3 b$ ). The red line is the cost functional based on LQG control designed from the identified model and the identified noise covariances. In addition to the cost functional $J$ the quantity $J_{k}=y_{k}^{2}+l u_{k}^{2}$ is displayed. The control is switched on at $t=400$, and a convection velocity of $U_{r}=3$ is chosen.

In figure 8, these two quantities are plotted as functions of $x$, as the dashed and full line, respectively. The main estimation error occurs upstream of the sensor, a region in which the two curves coincide. Physically, this corresponds to the failure of the sensor to accurately estimate information from upstream; the state downstream of the sensor, however, can be more easily estimated. In more quantitative terms, we introduce the ratio of the estimation-error standard deviation to the state standard deviation as a performance measure of the sensor. A ratio of $0 \%$ indicates an exact estimation of the state, whereas a value of $100 \%$ corresponds to the case where the estimator only returns the zero solution. This ratio is depicted in figure 9 for the two cases of moderate convection $U_{r}=2$ and large convection $U_{r}=3$, and it is evident that the state estimation becomes increasingly difficult upstream of the sensor. Based on these curves, we introduce the visibility length of the sensor as the maximum upstream distance from the sensor for which the relative estimation error is less than $50 \%$. Physically, this length gives a measure of how far upstream state information can be estimated within a reasonable tolerance. For $U_{r}=2$ this length is equal to 9.8, whereas for $U_{r}=3$ it is nearly half this value at 5.2. Figure 10 extends the visibility concept to even higher convection speeds and confirms the fact that estimation of upstream state information deteriorates rapidly as the convection speed increases.

This clearly demonstrates that state estimation in convection-dominated flows requires particular attention. To this end, we introduce an additional sensor $y_{s}$, referred to as a 'spy sensor', in an attempt to reduce the estimation error upstream. Since this is its only purpose, this additional sensor will not be included in the cost functional, in contrast to the first sensor. Its best placement is investigated by designing an optimal LQG control strategy based on the full-order system for each streamwise sensor position. The results of this parameter study are shown in figure 11, where 


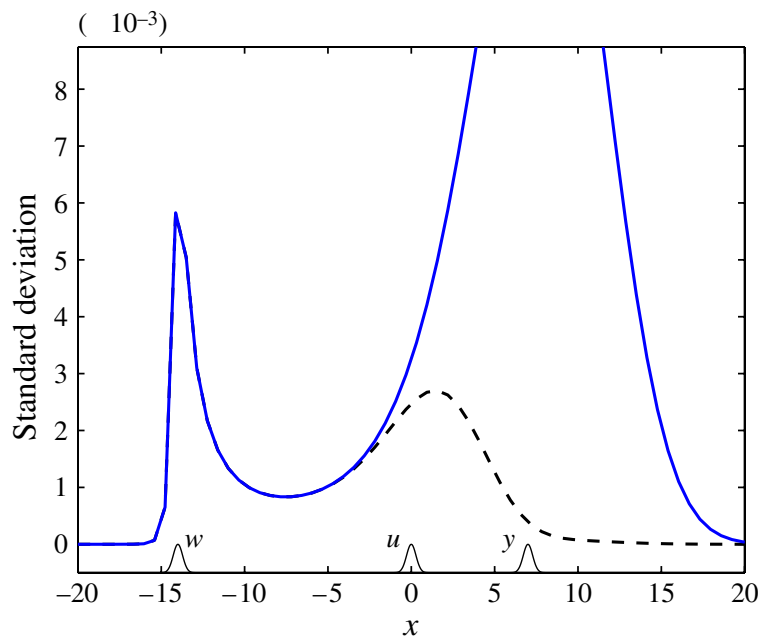

FIgURE 8. (Colour online) Standard deviation of the estimation error (dashed line) as a function of streamwise position $x$ and standard deviation of the state (full line) as a function of $x$. For both curves, the convection speed is $U_{r}=3$.

(a)

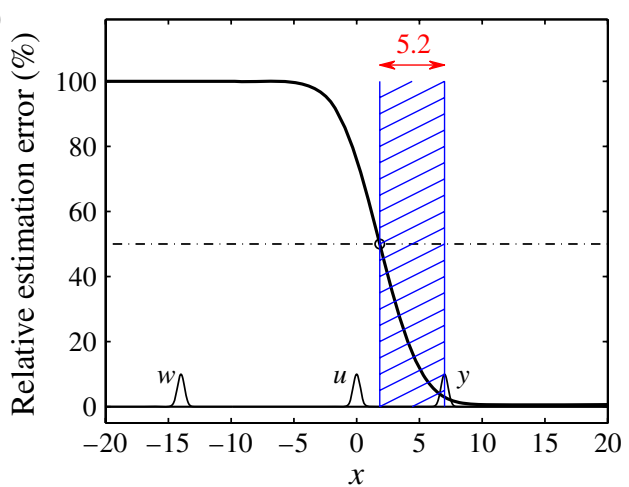

(b)

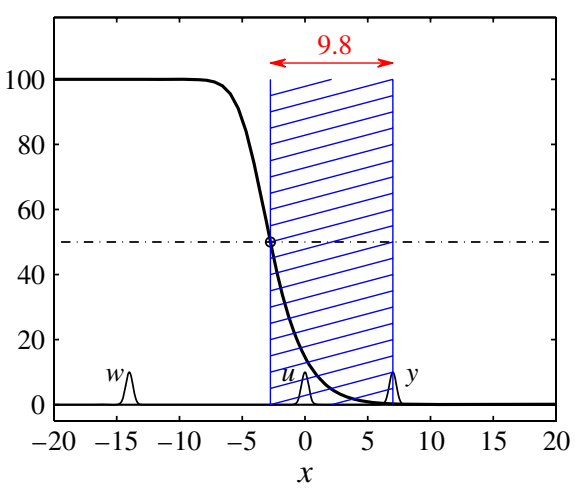

FIGURE 9. (Colour online) Ratio of the standard deviation of the estimation error to the standard deviation of the state as a function of streamwise position $x$ for a convection speed of (a) $U_{r}=3$ and (b) $U_{r}=2$. In both figures, the visibility length (see text) has been added.

the control efficiency, i.e. the ratio of the cost functionals with and without applied control, is evaluated for various spy sensor positions. The highest values of the control efficiency are achieved for a sensor placement upstream of the actuator, in accordance with figure 9, which showed poor state estimation in this region. In Hervé et al. (2010) a similar upstream sensor was used and referred to as a 'spy sensor'. In the present paper, this terminology is adopted and extended to the case of any convectiondominated system controlled by use of an upstream sensor. In addition, any sensor located sufficiently far downstream is referred to as an 'objective sensor'. In physical terms, the improvement in control efficiency from this spy sensor can be related to the more accurate estimation of incoming external noise. In other words, the spy sensor provides valuable information to the estimator on the incoming perturbations. Knowing 


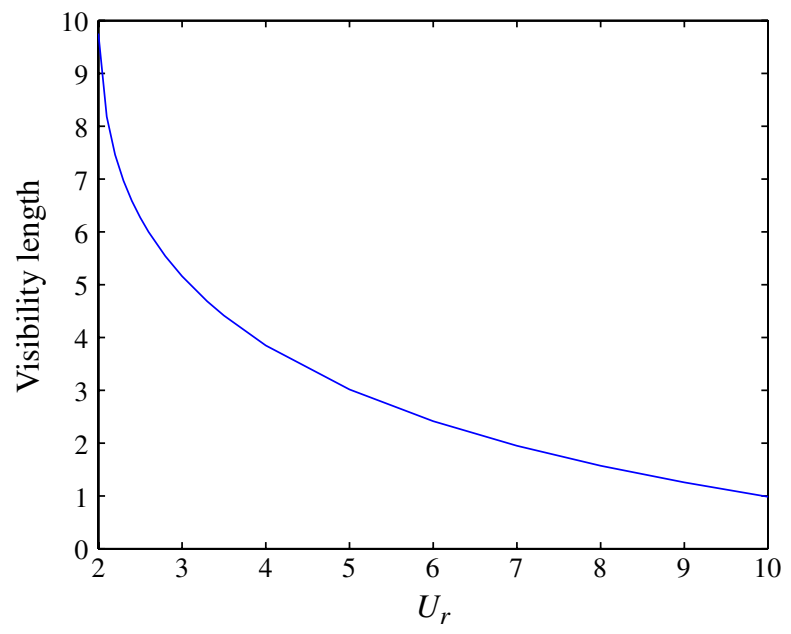

FIGURE 10. (Colour online) Visibility length as a function of the convection speed $U_{r}$ for the configuration displayed in figure 2 .

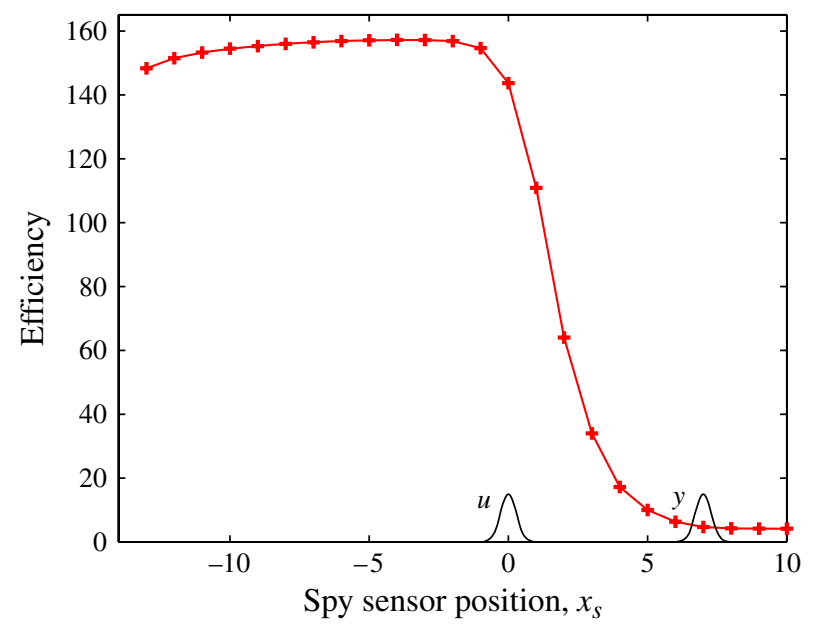

FIGURE 11. (Colour online) Efficiency of the optimal LQG control (based on the full-order system) as a function of the spy sensor position for the case of large convection $U_{r}=3$. The locations of the objective sensor and actuator are given in figure 2 .

these perturbations, it is then a lot easier to control them. Our findings are consistent with the studies of Barbagallo et al. (2012), who found that the best estimation results were obtained for the farthest upstream sensor.

\subsection{Failure and lack of robustness of the method}

So far, we have addressed the issues of a realistic control design (via subspace identification) and of an efficient control performance (via placement of a spy sensor). We now combine these two approaches into an identified control design using a spy sensor and consider the remaining issue of its robustness. 


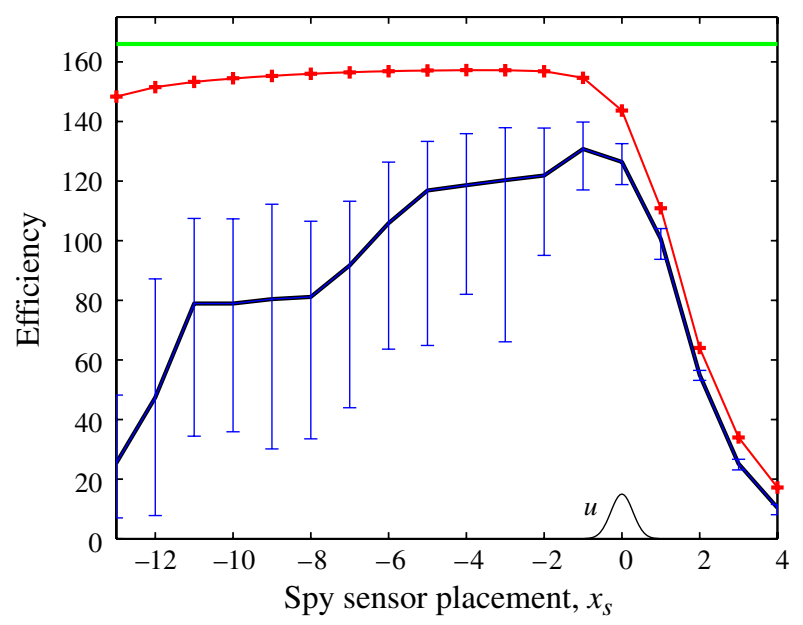

FIGURE 12. (Colour online) Control efficiency based on identified model versus position of the spy sensor $y_{s}$ for the case of large convection $U_{r}=3$. The identification follows the classical approach. Fifty realizations of identification and control were performed, and the average and one standard deviation are indicated. In practice, some unstable control cases were encountered. These curves are compared with the optimal LQG control designed from the full-order system (full line with plus symbols; red online) and the full-state control (horizontal line; green online). The actuator is located at $x_{u}=0$.

The control efficiency based on full-order LQG control, as shown in figure 11, will serve as the reference solution for the upper-bound or best-case control performance of our scheme. We therefore repeat the computations of the control efficiency based on the subspace identified system matrices and noise covariances. A remarkable sensitivity of the control performance to external noise sources, but also to userdefined parameters, such as the shift $i$ (see appendix A), the choice of subspace algorithm (MOESP, CVA or N4SID), the order of the identified model and the position of the spy sensor has been found. In practice, a small change in one of these parameters may even lead to an unstable control. A representative sample of our studies is shown in figure 12, where the average control efficiency over 50 realizations is displayed together with error bars. A marked drop in efficiency and sizable variations can be observed, yielding rather disappointing results compared to the reference full-order LQG case.

A possible reason for this sensitivity may be a weak and inaccurate link between the noise source very far upstream and the objective sensor downstream; this is sketched in figure 13. In general, the identification process establishes linear relations between inputs and outputs. In particular, the transfer function connecting known inputs and known outputs is rather easy to identify, for instance $u \rightarrow y$ (red online) in figure 13. Even an accurate model for $w \rightarrow y_{s}$ (blue online) can be found due to the stochastic nature of the subspace identification. The main difficulty, however, lies in the link between this upstream stochastic model $w \rightarrow y_{s}$ and the perturbation dynamics measured by the objective sensor $w \rightarrow y$ (dashed; green online). Identifying a model by subspace identification is akin to designing an estimator and it is therefore subject to the same visibility restrictions as discussed earlier. This also conforms with our intuition that, in convection-dominated flows, it becomes increasingly difficult to 


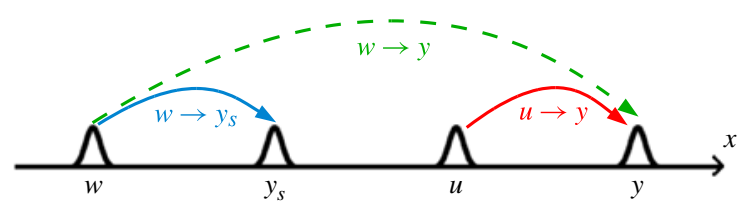

FIGURE 13. (Colour online) Sketch of the identification mechanism. Non-robustness stems from inaccuracies in the long-distance stochastic identification, shown dashed (green online).

estimate the perturbation dynamics when the unknown input $(w)$ moves further and further apart from the measured output $y$, as already demonstrated by introducing the concept of visibility. In fact, the success or failure of control depends on the longdistance stochastic identification represented as dashed (green online) in figure 13.

In summary, for convection-dominated flows the classical set-up, combining subspace identification and LQG control, results in a lack of robustness that renders this approach unusable for real-life applications.

\section{The feed-forward approach, a robust and efficient method for convection- dominated flows}

In this section a solution for the control of convection-dominated flows is presented. The approach is similar to the one developed in Hervé et al. (2012), although the practical implementation details are different. The general method is referred to as feed-forward in the control literature (Skogestad \& Postlethwaite 1996; Qin \& Badgwell 2003). The idea is to place a sensor $y_{s}$ sufficiently upstream to measure the incoming perturbations. This measurement $y_{s}$ is then used as a representation of the exact noise source $w$. As we will see, for convection-dominated flows this procedure is very robust with respect to system identification and it is close to the optimal LQG performance limit. Its realistic and robust characteristics should make it applicable to real experimental settings.

\subsection{Feed-forward identification and control}

We start by assuming that the noise perturbation $w_{k}$ is known. In this hypothetical situation, all inputs and outputs of the system are known, and a complete model can be identified by using data sequences of the input and output signals together with subspace identification techniques. From this model, the state vector can be accurately estimated at any time by simply exciting the model by known inputs.

In mathematical terms, an input-output model in state-space form is formulated according to

$$
\begin{aligned}
q_{k+1} & =\boldsymbol{A} q_{k}+\boldsymbol{B} u_{k}+\boldsymbol{B}_{w} w_{k}, \\
y_{k} & =\boldsymbol{C} q_{k}+v_{k},
\end{aligned}
$$

and the system matrices are determined by resorting to the MOESP identification technique. Based on this identified model, a full-state control may then be determined. At this point, the input $w$ is not taken into consideration for the design of the controller $\boldsymbol{K}$. The noise source $w$ is assumed to be known but it is not a control input. Rather, the control gain $\boldsymbol{K}$ is computed according to

$$
\boldsymbol{K}=-\left(l+\boldsymbol{B}^{H} \boldsymbol{T} \boldsymbol{B}\right)^{-1} \boldsymbol{B}^{H} \boldsymbol{T A},
$$




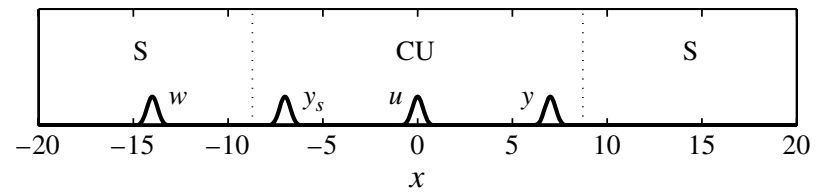

FIGURE 14. Sensor and actuator placement. The symbol $w$ represents the noise source at $x_{w}=-14, u$ is the actuator (the control) at $x_{u}=0, y$ is the objective sensor at $x_{y}=7$ and $y_{s}$ is the spy sensor placed at $x_{s}=-7$.

where $\boldsymbol{T}$ satisfies the discrete algebraic Riccati equation

$$
\boldsymbol{T}=\boldsymbol{A}^{H} \boldsymbol{T A}-\boldsymbol{A}^{H} \boldsymbol{T} \boldsymbol{B}\left(\boldsymbol{I}+\boldsymbol{B}^{H} \boldsymbol{S B}\right)^{-1} \boldsymbol{B}^{H} \boldsymbol{T A}+\boldsymbol{C}^{H} \boldsymbol{C} .
$$

The subspace identification process produces noise covariances $\boldsymbol{Q}, \boldsymbol{R}, \boldsymbol{S}$ (see $\S 3.1$ ) related to potentially unidentified input sources. In feed-forward mode for convectively dominated flows, this aspect of the estimation process is not exploited. With all input variables known, the estimator simply reads

$$
\tilde{q}_{k+1}=\boldsymbol{A} \tilde{q}_{k}+\boldsymbol{B} u_{k}+\boldsymbol{B}_{w} w_{k}
$$

and there is no need to compute and use the Kalman gain $\boldsymbol{L}$.

The feed-forward approach consists in adding a new spy sensor upstream of the actuator to measure the incoming noise (figure 14). Assuming that this measurement provides an accurate description of this noise, we may replace the true noise $w$ by the spy measurement $y_{s}$ in the above identification and control design algorithm. After substitution of the true noise $w$ by the proxy measurement $y_{s}$ from the spy sensor, the identified model reads

$$
\begin{aligned}
q_{k+1} & =\boldsymbol{A}^{\prime} q_{k}+\boldsymbol{B} u_{k}+\boldsymbol{B}_{s} y_{s, k}, \\
y_{k} & =\boldsymbol{C} q_{k} .
\end{aligned}
$$

(The matrix $\boldsymbol{A}^{\prime}$ in (4.5) differs from the matrix $\boldsymbol{A}$ in (4.1) due to the subtitution of $w$ by $y_{s}$. More details on this are given in $\S 6.2$ ). Note that all inputs to this model are known and it is therefore directly used to estimate the state. Based on this model, an optimal control gain can be computed by using the discrete Riccati equation given in (4.3).

\subsection{Efficiency of the method}

The feed-forward technique may then be implemented and evaluated. In figure 15 the efficiency of this control is plotted as a function of the spy sensor position $x_{s}$. First, a significantly improved robustness, as evidenced by the reduced standard deviation of the individual realizations, can be observed when compared to the stochastic identification and LQG control (figure 12). This improvement can be attributed to the absence of stochastic noise identification due to the substitution of $w$ by $y_{s}$. Any system identification algorithm would be able to produce a model linking known inputs $\left(u\right.$ and $\left.y_{s}\right)$ to a known output $y$. Even though subspace algorithms were found to be very robust and efficient, they do not constitute the only possible choice. In addition, the control based on the spy model is nearly as efficient as the optimal control based on the full-order matrices $\boldsymbol{A}, \boldsymbol{B}, \boldsymbol{C}, \boldsymbol{Q}, \boldsymbol{R}, \boldsymbol{S}$ (given by the full line with plus symbols; red online) and even close to full-state control (horizontal full line; green online). Figure 15 also illustrates that the control performance drops 


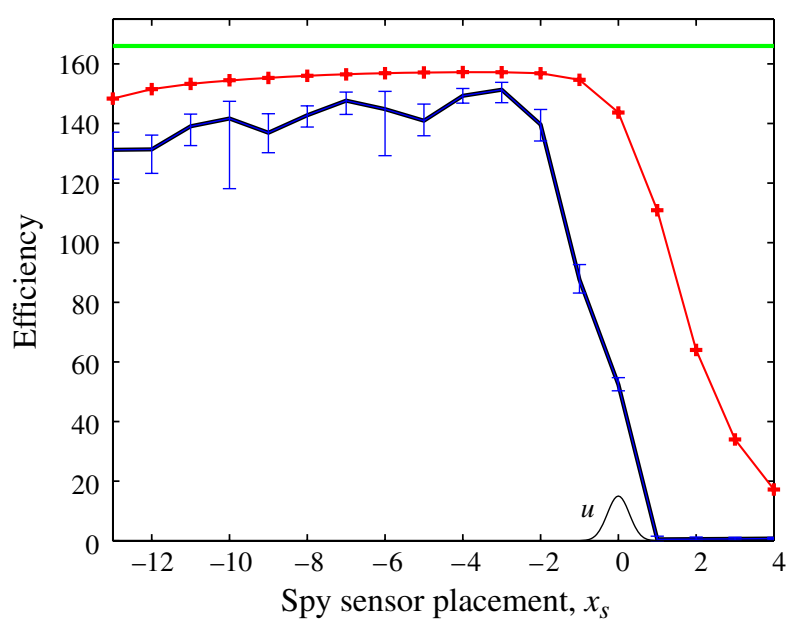

FIGURE 15. (Colour online) Control efficiency based on identified models versus position of the spy sensor $y_{s}$ for the case of large convection $U_{r}=3$. The identification follows the spy approach whereby the upstream sensor $y_{s}$ is considered as an input in the model. Fifty realizations of identification and control were performed, and the average and one standard deviation are indicated. In practice, all the controllers were stable. These curves are compared with the optimal LQG control designed from the full-order system (full line with plus symbols; red online) and the full-state control (horizontal line; green online). The actuator is located at $x_{u}=0$.

precipitously as the spy sensor approaches the actuator location $x_{u}=0$. Indeed, the spy sensor should provide upstream noise information and thus should not be corrupted by any action of the actuator $u$ (see $\S 6.2)$.

\subsection{Influence of convection}

The feed-forward identification and control are now applied to the Ginzburg-Landau equation for variable convection speeds $U_{r}$. For this model, the efficiency of the control, i.e. the relative reduction in the cost functional, is presented as a function of $U_{r}$ in figure 16. Two control design strategies are compared. The first strategy (lower full line with error bars; blue online) uses the feed-forward (spy) approach. In the identification process, the spy and the actuator are treated as inputs while the objective sensor downstream is taken as an output. In the control application, the objective sensor downstream is no longer needed and only the measurement from the spy is considered. The second strategy (full line with plus symbols; red online) is an LQG control based on the full-order system, with both sensors and the actuator. This strategy provides the best possible control for a given configuration and furnishes an upper bound for the system identified feed-forward control. As previously mentioned in $\$ 2.2$, the control performance becomes increasingly inefficient as the system becomes more convection-dominated. This appears clearly in the figure when considering the strong decrease in optimal efficiency (full line with plus symbols; red online) when the convection speed $U_{r}$ is increased. This is a general tendency, which holds true for any control strategy. In addition, by comparing the feed-forward control efficiency (full line with error bars; blue online) with this optimal upper bound, it is confirmed that the spy technique is almost optimal for large convection speed $U_{r}=3$. In contrast, although the feed-forward control is surprisingly effective 


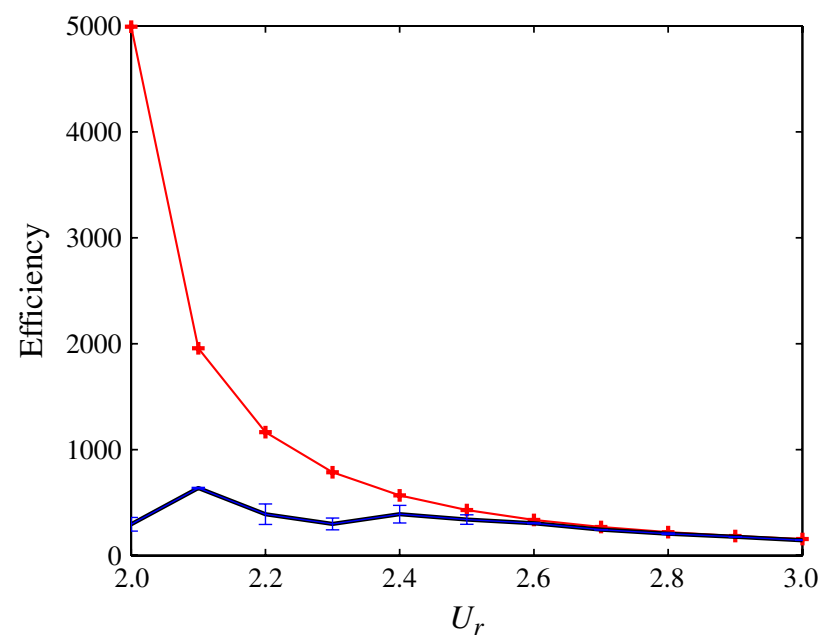

FIGURE 16. (Colour online) Control efficiency of the feed-forward approach as a function of the convection speed $U_{r}$ for the complex Ginzburg-Landau model. Fifty realizations of identification and control were performed; the average (full line with error bars; blue online) and one standard deviation are plotted. These curves are compared to the optimal LQG control designed from the full-order system (full line with plus symbols; red online). The spy sensor is placed at $x_{s}=-7$.

(with an efficiency of 500), it is far from optimality for moderate convection speed $U_{r}=2$. Two reasons for this non-optimality may be put forward. First, within the feed-forward control, the objective sensor downstream is not used for the estimation. However, it was shown in $\S 3.4$, using the associated visibility length, that for $U_{r}=2$ this sensor provides meaningful information regarding the state estimation. In addition, for moderate convection $U_{r}=2$, the theoretical upper bound of efficiency (full line with plus symbols; red online) is so high that the identification accuracy may become a limiting factor.

Hence, it has been seen that the feed-forward approach applied to amplifier flows is realistic, robust and efficient. In addition, the technique is close to optimal for noise amplifiers with sufficiently high convection. In the following, the technique is validated on a two-dimensional flow over a backward-facing step.

\section{Application to the flow over a two-dimensional backward-facing step}

The design of feed-forward control strategies based on subspace system identification has been introduced, illustrated and justified on the complex Ginzburg-Landau equation. It is now applied to the flow over a backward-facing step at $R e=350$. The three-dimensional stability of this flow was studied by Barkley, Gomes \& Henderson (2002) and a critical Reynolds number of 748 was found beyond which the flow becomes unstable. The first unstable mode is three-dimensional and localized in the recirculation bubble. More recently, this study has been extended to different step heights and the physical mechanisms leading to instability have been identified (Lanzerstorfer \& Kuhlmann 2012). In this investigation, it was also shown that the instability properties depend on the entrance length, and as a result the critical Reynolds number given by Barkley et al. (2002) was corrected from 748 to 714. In Blackburn, Barkley \& Sherwin (2008) the optimal disturbance and the transient growth 


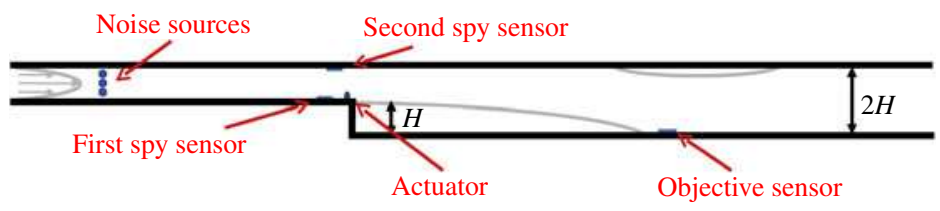

FIGURE 17. (Colour online) Sketch of the backward-facing step, displaying sensor and actuator positions; the two recirculation bubbles and the inlet Poiseuille profile are also indicated.

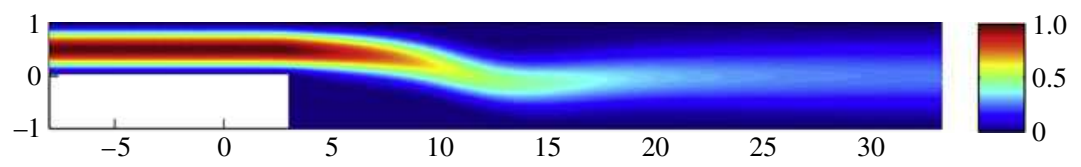

FIGURE 18. Base flow over a backward-facing step at $R e=350$. Colours correspond to the kinetic energy levels.

were examined. For a Reynolds number of 500, the maximum transient energy growth is $80 \times 10^{3}$.

Since a feed-forward controller has recently been applied and discussed in detail by Hervé et al. (2012) for the flow over a backward-facing step, the present section is kept brief. Its main objective is to validate the control technique developed in $\S 4$, to naturally extend it to the case of several spy sensors and to demonstrate its efficiency in the presence of multiple noise sources.

\subsection{System description}

A sketch of the configuration is presented in figure 17. The flow separates at the step corner and reattaches downstream at a distance equal to about nine step heights, in accordance with the computations of Barkley et al. (2002) and Blackburn et al. (2008). Under the selected flow conditions, a smaller recirculation bubble is also observed on the upper wall. These two bubbles induce strong shear stresses in the middle of the channel downstream of the step; this feature causes amplification of incoming perturbations via a Kelvin-Helmholtz instability. The objective of the control is to reduce this amplification. Although at $R e=350$, the flow is locally unstable, it is globally stable, since the local instability is convective in nature and any growing perturbation is rapidly transported downstream.

The steady base flow presented in figure 18 was obtained via a Newton-Raphson technique. The corresponding linear Navier-Stokes equations were then solved numerically with the finite-element software FreeFEM++. A linearized version of the Taylor-Hood algorithm was implemented. The domain was discretized in 42656 triangles, and a time step $\Delta t=0.03$ was chosen. No-slip boundary conditions at the wall, a parabolic velocity profile at the inlet and standard outflow boundary conditions were imposed. Three independent upstream noise sources (figure 17) were introduced and modelled by localized volumetric forces. The white noise was chosen to have a zero-mean unit-variance Gaussian probability density function. In order to control the incoming perturbations, an actuator was placed just upstream of the step corner $(x=1, y=0.12)$. The entire success of the feed-forward technique relies on the ability 


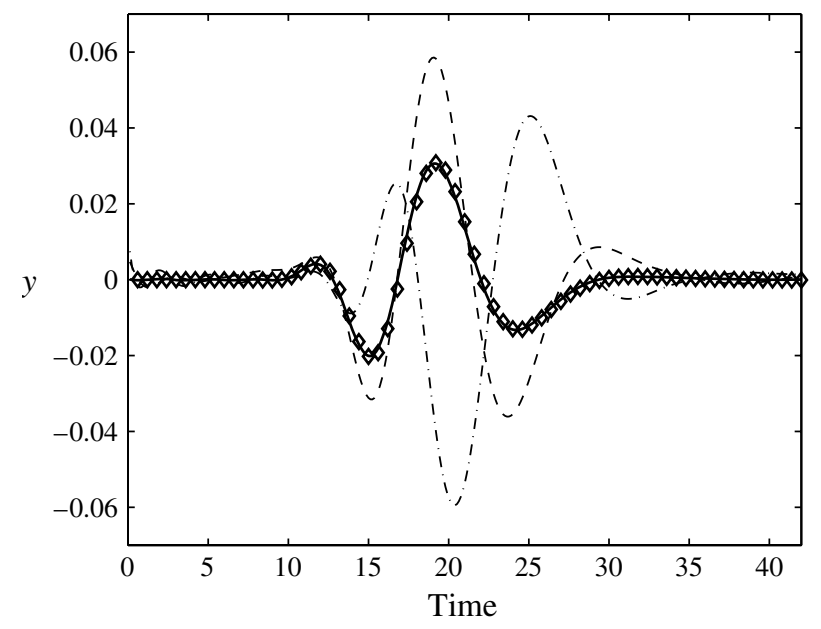

FIGURE 19. Comparison of the exact impulse response between the actuator $u$ and the objective sensor $y$ (diamonds) and the same impulse response determined from the identified model (full line). The identified impulse responses from the spy sensors to the objective sensor are also represented (dashed lines).

to accurately describe this complex noise structure. For that purpose two shear-stress spy sensors were introduced just upstream of the actuator, one on the upper wall and one on the lower wall. Since the flow over a backward-facing step is known to be particularly sensitive to any perturbation close to the step corner, one of the two spy sensors was placed close to this point. In addition, the second spy sensor, on the opposite wall, is able to capture incoming information that is not accessible to the first spy sensor. In general, for effective control the number of spy sensors depends on the complexity of the incoming perturbations that are to be modelled. Finally, the objective sensor was placed downstream near the lower reattachment point. All sensor measurements were corrupted by $10 \%$ of white noise.

\subsection{System identification}

Following the algorithm outlined in $\$ 3.1$ and in appendix $A$, a linear model is generated by subspace identification techniques. There are many possible choices for the actuator signal. In this work, the fluid is excited by two Gaussian white signals exiting from the actuators; other, more physical, choices are discussed in appendix B. At the same time, disturbances are generated by the noise sources, and the amplitudes of the actuator signals are chosen such that both the actuator signals and disturbances invoke about the same order of magnitude in the objective sensor signal. All signals used in the subspace identification algorithm have a length of 8000 time steps, and the shift parameter $i$ is 200. A model of order 19 is identified. This model expresses a causal link between the three upstream signals (two spy sensors and one actuator) and the objective sensor downstream. From this model, the three corresponding impulse responses can be determined and are displayed in figure 19 (full and dashed lines). For comparison, the exact impulse response from the actuator to the objective sensor is also displayed (diamonds). It is evident that the identified model captures very well the true system behaviour between the actuator and the objective sensor. From this model, the feed-forward compensator is designed as explained in $\S 4.1$. 


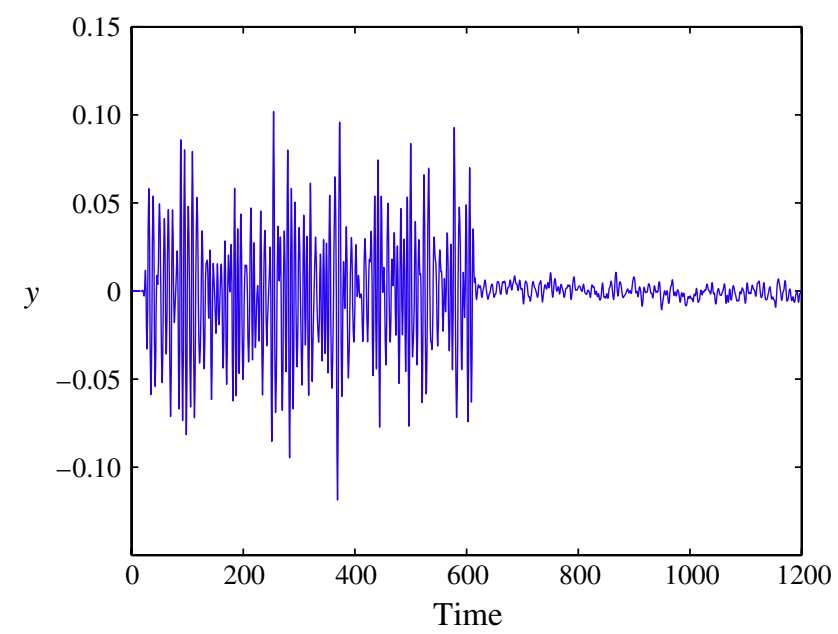

FIgURE 20. (Colour online) Signal measured at the objective sensor $y$. The control is activated at $t=600$.

\subsection{Results}

In figure 20, the signal measured by the objective sensor is plotted as a function of time. After 5000 time steps the control is activated, and an output reduction by a factor of approximately 10 is observed. In addition, this local reduction at the objective sensor location also results in a pronounced reduction of the flow perturbation energy downstream of the step. In figure $21(a, b)$, the time average of the perturbation norm is represented without and with control, respectively.

These results corroborate and further support the use of a feed-forward approach for convection-dominated systems. Based on the study of $\S 6.2$, we expect this control approach based on subspace identification to be nearly optimal. Moreover, the use of identification techniques makes the entire compensator design amenable to implementation in a real experiment.

\section{Theoretical basis of the feed-forward (spy) approach}

\subsection{A simple convection model}

We use a simplified model to further explain the optimal placement of the second sensor selected in $\$ 4.2$ in the context of the Ginzburg-Landau equation. It was observed in $\$ 3.4$ that state estimation and control efficiency improve significantly when the second sensor is placed upstream of the actuator. The model introduced below is intended to illustrate and clarify this finding. In addition, it will be demonstrated that the concept of observability is based on an asymptotic notion and it is thus inappropriate for describing sensor placement in convection-dominated flows.

To model convection-dominated phenomena in a small-order system, we base our model on a $6 \times 6$ shift operator, which, over each time step, advances the state vector $X=\left(x_{1}, x_{2}, x_{3}, x_{4}, x_{5}, x_{6}\right)^{\mathrm{T}}$ into a shifted vector $\left(0, x_{1}, x_{2}, x_{3}, x_{4}, x_{5}\right)^{\mathrm{T}}$. Note that this type of system can be obtained by discretizing an advection equation and choosing a unit Courant-Friedrichs-Lewy (CFL) number. A noise source is placed at the extreme upstream location, and an actuator is placed downstream. In mathematical terms, the 
(a)

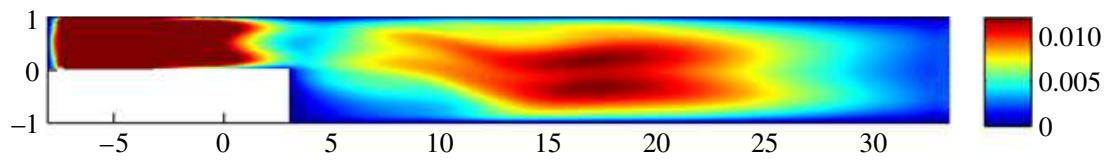

(b)

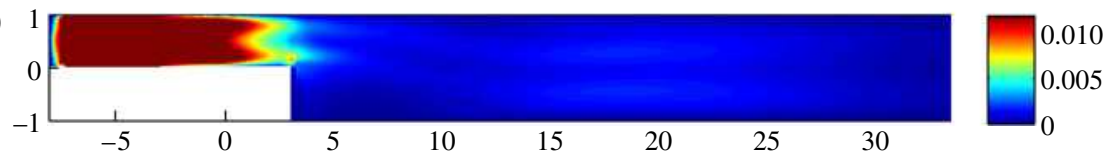

FIGURE 21. Time average of the perturbation norm for both $(a)$ the uncontrolled and $(b)$ the controlled cases.

full system is described by

$$
\begin{aligned}
X_{k+1} & =\boldsymbol{A} X_{k}+\boldsymbol{B}_{u} u_{k}+\boldsymbol{B}_{w} w_{k}, \\
y_{k} & =\boldsymbol{C} X_{k}+\mathbf{N} v_{k},
\end{aligned}
$$

where $X_{k}$ is the state vector, $u_{k}$ denotes the actuator input, $y_{k}$ stands for the sensor output, $w_{k}$ represents the stochastic excitation of the system (noise source) and $v_{k}$ is the measurement noise. We model $w_{k}$ and $v_{k}$ as uncorrelated Gaussian, white-noise sources of unit amplitude. In the above equation, $\boldsymbol{N}$ is a scalar that represents the measurement noise amplitude and it is assumed that $\|\boldsymbol{N}\| \ll 1$. According to this model, the system matrices are given as

$$
\boldsymbol{A}=\left(\begin{array}{llllll}
0 & 0 & 0 & 0 & 0 & 0 \\
1 & 0 & 0 & 0 & 0 & 0 \\
0 & 1 & 0 & 0 & 0 & 0 \\
0 & 0 & 1 & 0 & 0 & 0 \\
0 & 0 & 0 & 1 & 0 & 0 \\
0 & 0 & 0 & 0 & 1 & 0
\end{array}\right), \quad \boldsymbol{B}_{w}=\left(\begin{array}{l}
1 \\
0 \\
0 \\
0 \\
0 \\
0
\end{array}\right), \quad \boldsymbol{B}_{u}=\left(\begin{array}{l}
0 \\
0 \\
1 \\
0 \\
0 \\
0
\end{array}\right),
$$

and $\boldsymbol{C}$ is left to be defined later. As a prerequisite for efficient control, we proceed by computing an accurate estimate of the above system. For a given matrix $\boldsymbol{C}$, the governing equation for an estimator reads

$$
\begin{aligned}
\hat{X}_{k+1} & =\boldsymbol{A} \hat{X}_{k}+\boldsymbol{B}_{u} u_{k}+\boldsymbol{L}\left(y_{k}-\hat{y}_{k}\right), \\
\hat{y}_{k} & =\boldsymbol{C} \hat{X}_{k},
\end{aligned}
$$

where $\boldsymbol{L}$ is the optimal Kalman gain. The estimation error $e_{k} \equiv X_{k}-\hat{X}_{k}$ then satisfies

$$
e_{k+1}=(\boldsymbol{A}-\boldsymbol{L C}) e_{k}+\boldsymbol{B}_{w} w_{k}-\boldsymbol{L} \boldsymbol{N} v_{k} .
$$

In the following we wish to determine an optimal sensor placement that minimizes this estimation error.

\subsubsection{Sensor placement}

Two possible choices for the sensor placement are considered. The first one corresponds to a sensor located far downstream, i.e.

$$
\boldsymbol{C}=\boldsymbol{C}_{1} \equiv\left(\begin{array}{llllll}
0 & 0 & 0 & 0 & 0 & 1
\end{array}\right) .
$$


This choice (downstream of the actuator) is often preferred in the flow control literature (Bagheri et al. 2009b; Barbagallo 2011; Barbagallo et al. 2012), and may be justified by the argument that it yields high observability. In addition, if the control objective is to minimize the norm of $y$, which is often the case in practice, placing the sensor downstream of the actuator appears intuitive, since it guarantees a non-zero transfer function from $u$ to $y$. However, it will be seen below that, for convection-dominated systems, this choice is extremely disadvantageous for the state estimation.

It can be easily verified by computing the rank of the observability matrix $\boldsymbol{O b}_{1}$ that the system $\left(\boldsymbol{A}, \boldsymbol{C}_{1}\right)$ is observable (Zhou et al. 1996). We obtain the full-rank matrix

$$
\boldsymbol{O} \boldsymbol{b}_{1}=\left(\begin{array}{c}
\boldsymbol{C}_{1} \\
\boldsymbol{C}_{1} \boldsymbol{A} \\
\boldsymbol{C}_{1} \boldsymbol{A}^{2} \\
\boldsymbol{C}_{1} \boldsymbol{A}^{3} \\
\boldsymbol{C}_{1} \boldsymbol{A}^{4} \\
\boldsymbol{C}_{1} \boldsymbol{A}^{5}
\end{array}\right)=\left(\begin{array}{llllll}
0 & 0 & 0 & 0 & 0 & 1 \\
0 & 0 & 0 & 0 & 1 & 0 \\
0 & 0 & 0 & 1 & 0 & 0 \\
0 & 0 & 1 & 0 & 0 & 0 \\
0 & 1 & 0 & 0 & 0 & 0 \\
1 & 0 & 0 & 0 & 0 & 0
\end{array}\right)
$$

Recall that a system is observable if and only if all the eigenvalues of the matrix $\boldsymbol{A}-\boldsymbol{L C}$ can be placed arbitrarily by choosing the coefficients of $\boldsymbol{L}$. Defining $\boldsymbol{L}=\left(l_{1}, l_{2}, l_{3}, l_{4}, l_{5}, l_{6}\right)^{\mathrm{T}}$ in the system above, we arrive at $\operatorname{det}(\lambda \boldsymbol{I}-\boldsymbol{A}+\boldsymbol{L C})=$ $\lambda^{6}+l_{6} \lambda^{5}+l_{5} \lambda^{4}+l_{4} \lambda^{3}+l_{3} \lambda^{2}+l_{2} \lambda+l_{1}$, which shows that we have full control over the eigenvalues of $\boldsymbol{A}-\boldsymbol{L C}$ and therefore over the long-time dynamics of the estimation error, governed by (6.4). However, a performing estimator should also provide an approximation of the state on a short time scale - in particular, in strongly convective systems where perturbations are quickly swept downstream and measured information quickly becomes non-pertinent. Tuning the eigenvalues is insufficient for guaranteeing optimal estimator performance on account of the presence of transient effects; we also have to consider the corresponding eigenvectors of $\boldsymbol{A}-\boldsymbol{L C}$. This is due to the fact that the matrix $\boldsymbol{A}$ describes a convective process and is therefore highly non-normal (Chomaz 2005).

From a physical point of view, it is clear that information from a downstream sensor is futile since it is immediately swept away. This is further corroborated when computing the Kalman gain $L_{1}$ in the presence of stochastic noise. This gain is governed by the discrete Riccati equation

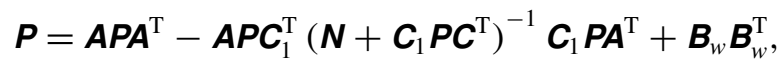

$$
\begin{aligned}
& \boldsymbol{L}_{1}=\boldsymbol{A} \boldsymbol{P} \boldsymbol{C}_{1}^{\mathrm{T}}\left(\boldsymbol{N}+\boldsymbol{C}_{1} P \boldsymbol{C}_{1}^{\mathrm{T}}\right)^{-1} .
\end{aligned}
$$

For a far-downstream sensor location $\boldsymbol{C}_{1}$, it can be verified that the optimal Kalman gain $L_{1}$ tends to zero for vanishing $\boldsymbol{N}$, i.e.

$$
\lim _{N \rightarrow 0} L_{1}=0 .
$$

This limit supports mathematically the intuitive argument above, namely, that input from $y$ does not enter the estimation of the state given by (6.3). In other words, this downstream sensor could simply be removed without any consequences for the control performance. 
In contrast, we now consider a configuration where the sensor is placed at a very upstream position so that

$$
\boldsymbol{C}=\boldsymbol{C}_{2} \equiv\left(\begin{array}{llllll}
0 & 1 & 0 & 0 & 0 & 0
\end{array}\right) .
$$

Note that the system $\left(\boldsymbol{A}, \boldsymbol{C}_{2}\right)$ is clearly not observable, since

$$
\boldsymbol{O b}_{2}=\left(\begin{array}{c}
\boldsymbol{C}_{2} \\
\boldsymbol{C}_{2} \boldsymbol{A} \\
\boldsymbol{C}_{2} \boldsymbol{A}^{2} \\
\boldsymbol{C}_{2} \boldsymbol{A}^{3} \\
\boldsymbol{C}_{2} \boldsymbol{A}^{4} \\
\boldsymbol{C}_{2} \boldsymbol{A}^{5}
\end{array}\right)=\left(\begin{array}{llllll}
0 & 1 & 0 & 0 & 0 & 0 \\
1 & 0 & 0 & 0 & 0 & 0 \\
0 & 0 & 0 & 0 & 0 & 0 \\
0 & 0 & 0 & 0 & 0 & 0 \\
0 & 0 & 0 & 0 & 0 & 0 \\
0 & 0 & 0 & 0 & 0 & 0
\end{array}\right)
$$

Nonetheless, this sensor placement can lead to a very accurate state estimation. Intuitively, for a sensor placed sufficiently upstream, all components of the current state vector $X_{k}$ have previously been measured by the sensor before being convected downstream. As before, the Kalman gain may be obtained from a discrete Riccati equation, with $\boldsymbol{C}=\boldsymbol{C}_{2}$. It now reads

$$
\lim _{N \rightarrow 0} \boldsymbol{L}_{2}=\left(\begin{array}{l}
0 \\
0 \\
1 \\
0 \\
0 \\
0
\end{array}\right) .
$$

Consequently, the estimation error given by (6.4) is

$$
e_{k}=\left(\begin{array}{c}
w_{k-1} \\
w_{k-2} \\
0 \\
0 \\
0 \\
0
\end{array}\right) \text {. }
$$

We observe that the estimated state vector is exact (zero estimation error) everywhere downstream of the sensor location. This demonstrates that, in convection-dominated flows, valuable information on the future downstream state can be obtained from an upstream sensor; this is also consistent with our earlier study of the visibility length in §3.4. Even though the system becomes more observable by placing the sensor further downstream, the drastic decrease in control performance makes this choice highly ineffective. This also confirms that conventional observability measures yield misleading suggestions regarding optimal sensor placement, especially in convectiondominated (and, more generally, non-normal) systems.

\section{2. $L Q G$ control design and feed-forward approach: a comparison}

In this section a theoretical comparison between the feed-forward approach and the optimal LQG framework is presented, and conditions are derived under which these 


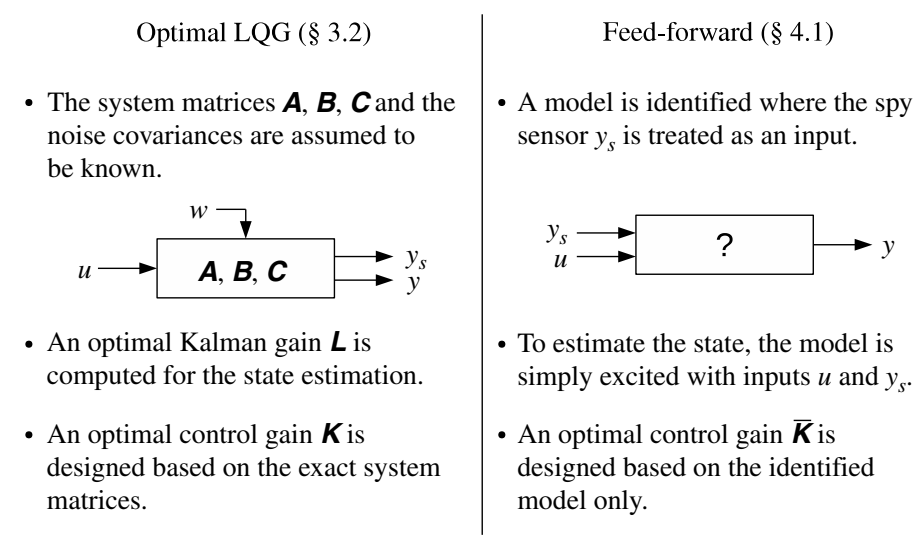

FIGURE 22. Computational steps of the LQG and feed-forward procedures. Section 6.2 gives a theoretical comparison of these two control frameworks.

two control strategies are equivalent. In this manner, we establish situations for which the feed-forward technique is optimal. The configuration under study is very general; the flow is convection-dominated with one actuator $u$, one upstream spy sensor $y_{s}$ and one downstream objective sensor $y_{o b}$. Flow over a backward-facing step, as in $\S 5$, is a typical example, but more generally we consider any convection-dominated flow such as channel flows, homogeneous jet flows or flows over streamlined aerofoils. The optimal, but unrealistic, LQG algorithm is described in the left column of figure 22 . It is compared with the realistic feed-forward technique summarized in the right column of figure 22. Rather than focusing on the very specific accuracy of the system identification stage, which might be improved by simply increasing the length of the input-output data sequences, it is assumed that the systems are identified exactly. Hence, the last two steps of the techniques are compared and the analysis naturally divides into the study of estimation processes and of control gains.

\subsubsection{Estimation}

In this section, the state estimation stages of both techniques are compared. It has already been argued in $\S \S 3.4$ and 6.1 that the downstream measurement from the objective sensor does not contribute to state estimation in convection-dominated flows. The optimal Kalman gain $\boldsymbol{L}$ is therefore designed assuming an estimation from the spy sensor $y_{s}$ only. As detailed in $\S 3.2$, the Kalman estimator of the optimal LQG method reads

$$
\begin{aligned}
\tilde{X}_{k+1} & =\left(\boldsymbol{A}-\boldsymbol{L} \boldsymbol{C}_{s}\right) \widetilde{X}_{k}+\boldsymbol{B} u_{k}+\boldsymbol{L} y_{s, k}, \\
\tilde{y}_{k} & =\boldsymbol{C}_{o b} \widetilde{X}_{k},
\end{aligned}
$$

where $\boldsymbol{C}_{s}$ and $\boldsymbol{C}_{o b}$ are respectively the spy and objective measurement matrices and $\widetilde{X}$ is the estimated state. The first equation (6.13a) has been generated from system (3.6) by adding an $s$ subscript (for spy) to $y, \tilde{y}$ and $\boldsymbol{C}$. The second equation $(6.13 b)$ is a measurement equation that provides an estimate of the objective sensor measurement from the estimated state. The equivalent measurement equation for the spy sensor is not explicitly written. The Kalman estimator written in (6.13) is a linear system with two inputs $u$ and $y_{s}$ and one output $\tilde{y}$. In the following, it is claimed and 

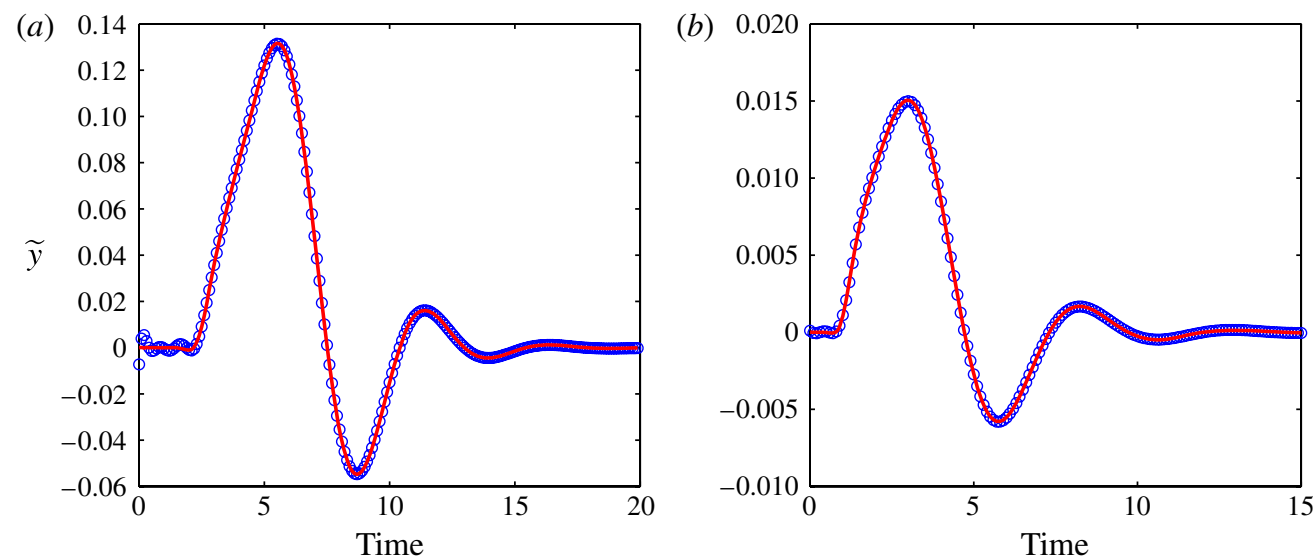

FIGURE 23. (Colour online) Impulse response generated from $(a)$ an impulse applied to $y_{s}$ and measured in $\tilde{y}$ and $(b)$ an impulse applied to $u$ and measured in $\tilde{y}$. The full lines (red online) pertain to the optimal Kalman estimator, whereas the circles (blue online) pertain to the feed-forward identified model.

numerically verified that this Kalman estimator is precisely the identified model of the feed-forward method outlined on the right of figure 22 .

In the feed-forward identification procedure (stage one in the right column of figure 22), a linear system is sought with inputs $u$ and $y_{s}$ and output $\tilde{y}$ such that the output error $\|\tilde{y}-y\|$ is minimized. This property is already verified by the estimator (6.13). Assuming that the Kalman estimator is the only system minimizing the output error $\|\tilde{y}-y\|$, we may conclude that the feed-forward procedure aims at identifying the Kalman estimator. In other words, the identification procedure consists in identifying the matrices $\boldsymbol{A}^{\prime}=\boldsymbol{A}-\boldsymbol{L C}, \boldsymbol{B}, \boldsymbol{L}$ and $\boldsymbol{C}_{o b}$, which precisely coincides with the determination of the Kalman estimator. Note that a similar property is the basis of other control schemes such as the observer/Kalman filter identification (OKID) procedure (Juang et al. 1991).

At this point, it is worth comparing the input-output dynamics of the feed-forward identified model and of the Kalman estimator, as displayed in figure 23. The system under consideration is the Ginzburg-Landau model for large convection $U_{r}=3$, as described in $\$ 2$, with the sensor-actuator configuration sketched in figure 14 . The impulse responses of each system are represented in figure 23: it is clear that the feed-forward model is identical to the optimal Kalman estimator. Hence, treating the measurement $y_{s}$ as an input in the identification stage coincides with the computation of the Kalman estimator, and thus the estimation stages in the two techniques are identical.

\subsubsection{Control gain}

The equivalence of the estimation process is now contrasted with the design of the control gain for the two methods, because, in the end, the product of control gain and estimated state will determine the final control law. The difference in control gains stems from the fact that in the feed-forward approach the gain $\overline{\boldsymbol{K}}$ is determined from a discrete Riccati equation involving $\boldsymbol{A}^{\prime}=\boldsymbol{A}-\boldsymbol{L} \boldsymbol{C}_{s}$, whereas in the LQG approach a discrete Riccati equation involving $\boldsymbol{A}$ is used. The consequences of such an observation are given below. In terms of equations, if the objective to be minimized is given by $\mathscr{E}\left(\|y\|^{2}+l\|u\|^{2}\right)$, the LQG control gain $\boldsymbol{K}$ is given by the discrete Riccati equation 
involving $\boldsymbol{A}$,

$$
\begin{aligned}
& \boldsymbol{S}=\boldsymbol{A}^{H}\left(\boldsymbol{S}-\boldsymbol{S B}\left(l+\boldsymbol{B}^{H} \boldsymbol{S} \boldsymbol{B}\right)^{-1} \boldsymbol{B}^{H} \boldsymbol{S}\right) \boldsymbol{A}+\boldsymbol{C}_{o b}{ }^{H} \boldsymbol{C}_{o b}, \\
& \boldsymbol{K}=-\left(l+\boldsymbol{B}^{H} \boldsymbol{S B}\right)^{-1} \boldsymbol{B}^{H} \boldsymbol{S A},
\end{aligned}
$$

while the feed-forward (spy) control gain $\overline{\boldsymbol{K}}$ is determined by the discrete Riccati equation based on $\boldsymbol{A}^{\prime}=\boldsymbol{A}-\boldsymbol{L} \boldsymbol{C}_{s}$,

$$
\begin{aligned}
& \overline{\boldsymbol{S}}=\left(\boldsymbol{A}-\boldsymbol{L} \boldsymbol{C}_{s}\right)^{H}\left(\overline{\boldsymbol{S}}-\overline{\boldsymbol{S}} \boldsymbol{B}\left(l+\boldsymbol{B}^{H} \overline{\boldsymbol{S}} \boldsymbol{B}\right)^{-1} \boldsymbol{B}^{H} \overline{\boldsymbol{S}}\right)\left(\boldsymbol{A}-\boldsymbol{L} \boldsymbol{C}_{s}\right)+\boldsymbol{C}_{o b}{ }^{H} \boldsymbol{C}_{o b}, \\
& \overline{\boldsymbol{K}}=-\left(l+\boldsymbol{B}^{H} \overline{\boldsymbol{S}} \boldsymbol{B}\right)^{-1} \boldsymbol{B}^{H} \overline{\boldsymbol{S}}\left(\boldsymbol{A}-\boldsymbol{L} \boldsymbol{C}_{s}\right) .
\end{aligned}
$$

Based on these two equations, we proceed by comparing the two control signals $u_{k}=\boldsymbol{K} \hat{X}_{k}$ and $\bar{u}_{k}=\overline{\boldsymbol{K}} \hat{X}_{k}$.

To this end, the estimator $(6.13 a)$ is rewritten in canonical Kalman form (Zhou et al. 1996). This form is obtained via a change of variables, and it can be shown that it is mathematically equivalent to $(6.13 a)$. This canonical Kalman form reads:

$$
\begin{aligned}
&\left(\begin{array}{c}
\hat{X}_{c o / k+1} \\
\hat{X}_{c \bar{o} / k+1} \\
\hat{X}_{\bar{c} o / k+1} \\
\hat{X}_{\overline{c o} / k+1}
\end{array}\right)=\left(\begin{array}{cccc}
\boldsymbol{A}_{c o} & 0 & \boldsymbol{A}_{13} & 0 \\
\boldsymbol{A}_{21} & \boldsymbol{A}_{c \bar{o}} & \boldsymbol{A}_{23} & \boldsymbol{A}_{24} \\
0 & 0 & \boldsymbol{A}_{\bar{c} o} & 0 \\
0 & 0 & \boldsymbol{A}_{43} & \boldsymbol{A}_{\overline{c o}}
\end{array}\right)\left(\begin{array}{c}
\hat{X}_{c o / k} \\
\hat{X}_{c \bar{o} / k} \\
\hat{X}_{\overline{c o} / k} \\
\hat{X}_{\overline{c o} / k}
\end{array}\right)+\left(\begin{array}{cc}
\boldsymbol{B}_{c o} & \overline{\boldsymbol{L}}_{c o} \\
\boldsymbol{B}_{c \bar{o}} & \overline{\boldsymbol{L}}_{c \bar{o}} \\
0 & 0 \\
0 & 0
\end{array}\right)\left(\begin{array}{c}
u_{k} \\
y_{s, k}-\hat{y}_{s, k}
\end{array}\right) \\
& \hat{y}_{s, k}=\left(\begin{array}{llll}
\boldsymbol{C}_{s, c o} & 0 & \boldsymbol{C}_{s, \overline{c o}} & 0
\end{array}\right)\left(\begin{array}{l}
\hat{X}_{c o / k} \\
\hat{X}_{c \bar{o} / k} \\
\hat{X}_{\bar{c} o / k} \\
\hat{X}_{\overline{c o} / k}
\end{array}\right)
\end{aligned}
$$

The second equation (6.16b) gives an optimal estimate of the spy sensor measurement. The equivalent equation for the objective sensor is not considered. The subscript co denotes controllable and observable components, $c \bar{o}$ stands for controllable but unobservable components, $\bar{c} o$ represents uncontrollable but observable components, and $\overline{c o}$ means both uncontrollable and unobservable components. By definition the observability and controllability matrix of the canonical Kalman form, i.e.

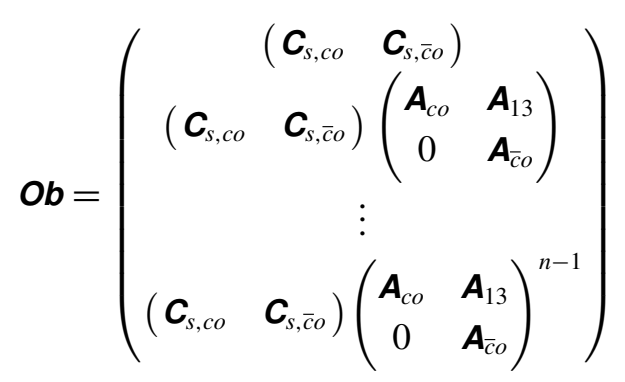

and

$\boldsymbol{C t r}=\left(\left(\begin{array}{cc}\boldsymbol{B}_{c o} & \overline{\mathbf{L}}_{c o} \\ \boldsymbol{B}_{c \bar{o}} & \overline{\mathbf{L}}_{c \bar{o}}\end{array}\right)\left(\begin{array}{cc}\boldsymbol{A}_{c o} & 0 \\ \boldsymbol{A}_{21} & \boldsymbol{A}_{c \bar{o}}\end{array}\right)\left(\begin{array}{cc}\boldsymbol{B}_{c o} & \overline{\boldsymbol{L}}_{c o} \\ \boldsymbol{B}_{c \bar{o}} & \overline{\boldsymbol{L}}_{c \bar{o}}\end{array}\right) \ldots\left(\begin{array}{cc}\boldsymbol{A}_{c o} & 0 \\ \boldsymbol{A}_{21} & \boldsymbol{A}_{c \bar{o}}\end{array}\right)^{n-1}\left(\begin{array}{cc}\boldsymbol{B}_{c o} & \overline{\mathbf{L}}_{c o} \\ \boldsymbol{B}_{c \bar{o}} & \overline{\boldsymbol{L}}_{c \bar{o}}\end{array}\right)\right)(6.1$

have full column and row rank, respectively. The same decomposition into (un)controllable and (un)observable components can be applied to both control gains $\boldsymbol{K}$ 
and $\overline{\boldsymbol{K}}$ according to $\boldsymbol{K}=\left(\begin{array}{lllll}\boldsymbol{K}_{c o} & \boldsymbol{K}_{c \bar{o}} & \boldsymbol{K}_{\overline{c o}} & \boldsymbol{K}_{\overline{c o}}\end{array}\right)$ and $\overline{\boldsymbol{K}}=\left(\begin{array}{llll}\overline{\boldsymbol{K}}_{c o} & \overline{\boldsymbol{K}}_{c \bar{o}} & \overline{\boldsymbol{K}}_{\bar{c} o} & \overline{\boldsymbol{K}}_{\overline{c o}}\end{array}\right)$. In what follows we compare the individual components of the two control gains and interpret their significance. Without loss of generality, we choose $\hat{X}_{0}=0$ as the initial estimated state, from which it follows that both $\hat{X}_{\overline{c o} / k}$ and $\hat{X}_{\overline{c o} / k}$ are zero for all $k$. As a result, the control inputs may be expanded as

$$
\begin{aligned}
& u_{k}=\boldsymbol{K}_{c o} \hat{X}_{c o / k}+\boldsymbol{K}_{c \bar{D}} \hat{X}_{c \bar{o} / k}+\boldsymbol{K}_{\overline{c o}} \hat{X}_{\bar{c} o / k}+\boldsymbol{K}_{\overline{c o}} \hat{X}_{\overline{c o} / k}=\left(\begin{array}{llll}
\boldsymbol{K}_{c o} & \boldsymbol{K}_{c \bar{o}} & 0 & 0
\end{array}\right) \hat{X}_{k}, \\
& \bar{u}_{k}=\overline{\boldsymbol{K}}_{c o} \hat{X}_{c o / k}+\overline{\boldsymbol{K}}_{c \bar{o}} \hat{X}_{\bar{c} \bar{c} / k}+\overline{\boldsymbol{K}}_{\overline{c o}} \hat{X}_{\bar{c} o / k}+\overline{\boldsymbol{K}}_{\overline{c o}} \hat{X}_{\overline{\bar{c}} / k}=\left(\begin{array}{llll}
\overline{\boldsymbol{K}}_{c o} & \overline{\boldsymbol{K}}_{\bar{c} \bar{o}} & 0 & 0
\end{array}\right) \hat{X}_{k} .
\end{aligned}
$$

Note that neither the LQG control $u_{k}$ nor the feed-forward (spy) control $\bar{u}_{k}$ depend on the uncontrollable part $\bar{c}$ of the estimator. This uncontrollable part of the estimator represents the subspace of the state that cannot be estimated at all, and it appears appropriate that neither control depends on this input. What remains to be seen is

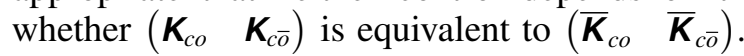

The following analysis rests on the assumption that there is no influence of the actuator on the upstream spy sensor. This may be expressed by a zero impulse response from $u$ to $y_{s}$, which appears reasonable for convection-dominated flows. Under this assumption, we form the product of the observability matrix $\mathbf{O} b$ and the control input matrix $\left(\boldsymbol{B}_{c o}^{\mathrm{T}} \quad 0\right)^{\mathrm{T}}$ to obtain

$$
\boldsymbol{O b}\left(\begin{array}{c}
\boldsymbol{B}_{c o} \\
0
\end{array}\right)=\left(\begin{array}{c}
\left(\begin{array}{cc}
\boldsymbol{C}_{s, c o} & \boldsymbol{C}_{s, \overline{c o}}
\end{array}\right) \\
\left(\begin{array}{cc}
\boldsymbol{C}_{s, c o} & \boldsymbol{C}_{s, \bar{c} o}
\end{array}\right)\left(\begin{array}{cc}
\boldsymbol{A}_{c o} & \boldsymbol{A}_{13} \\
0 & \boldsymbol{A}_{\overline{c o}}
\end{array}\right) \\
\vdots \\
\left(\begin{array}{cc}
\boldsymbol{C}_{s, c o} & \boldsymbol{C}_{s, \bar{c} o}
\end{array}\right)\left(\begin{array}{cc}
\boldsymbol{A}_{c o} & \boldsymbol{A}_{13} \\
0 & \boldsymbol{A}_{\overline{c o}}
\end{array}\right)^{n-1}
\end{array}\right)\left(\begin{array}{c}
\boldsymbol{B}_{c o} \\
0
\end{array}\right)=\left(\begin{array}{c}
\boldsymbol{C}_{s, c o} \boldsymbol{B}_{c o} \\
\boldsymbol{C}_{s, c o} \boldsymbol{A}_{c o} \boldsymbol{B}_{c o} \\
\vdots \\
\boldsymbol{C}_{s, c o} \boldsymbol{A}_{c o}^{n-1} \boldsymbol{B}_{c o}
\end{array}\right) .
$$

Since the elements $\boldsymbol{C}_{s, c o} \boldsymbol{B}_{c o}, \boldsymbol{C}_{s, c o} \boldsymbol{A}_{c o} \boldsymbol{B}_{c o}, \ldots, \boldsymbol{C}_{s, c o} \boldsymbol{A}_{c o}^{n-1} \boldsymbol{B}_{c o}$ represent the first $n$ values of the discrete impulse response (Markov parameters) from $u$ to $y_{s}$, and since this impulse response is assumed to be identically zero, we conclude that

$$
\mathbf{O b}\left(\begin{array}{c}
\boldsymbol{B}_{c o} \\
0
\end{array}\right)=0 \text {. }
$$

Owing to the full column rank of $\boldsymbol{O} \boldsymbol{b}$, one has $\boldsymbol{B}_{c o}=0$. A careful reorganization and partitioning of the two discrete Riccati equations (6.14) and (6.15), using $\boldsymbol{B}_{c o}=0$ as well as the block structure of (6.16), yields

$$
\begin{aligned}
& \boldsymbol{K}_{\bar{c}}=-\left(l+\boldsymbol{B}_{c \bar{o}}^{H} \boldsymbol{S}_{\bar{c} \overline{\bar{o}}} \boldsymbol{B}_{c \bar{o}}\right)^{-1} \boldsymbol{B}_{c \bar{o}}^{H} \boldsymbol{S}_{c \bar{o}} \boldsymbol{A}_{\bar{c} \bar{\theta}}, \\
& \overline{\boldsymbol{K}}_{\bar{c}}=-\left(l+\boldsymbol{B}_{c \bar{o}}^{H} \overline{\boldsymbol{S}}_{\bar{c} \bar{b}} \boldsymbol{B}_{c \bar{b}}\right)^{-1} \boldsymbol{B}_{c \bar{o}}^{H} \overline{\boldsymbol{S}}_{\bar{c}} \boldsymbol{A}_{c \bar{o}}
\end{aligned}
$$

where $\boldsymbol{S}_{c \bar{o}}$ and $\overline{\boldsymbol{S}}_{\bar{c} \bar{b}}$ are solutions of

$$
\begin{aligned}
& \boldsymbol{S}_{c \bar{o}}=\boldsymbol{A}_{c \bar{o}}^{H}\left(\boldsymbol{S}_{c \bar{o}}-\boldsymbol{S}_{c \bar{o}} \boldsymbol{B}_{c \bar{o}}\left(l+\boldsymbol{B}_{c \bar{c}}^{H} \boldsymbol{S}_{c \bar{o}} \boldsymbol{B}_{c \bar{o}}\right)^{-1} \boldsymbol{B}_{c \bar{D}}^{H} \boldsymbol{S}_{c \bar{o}}\right) \boldsymbol{A}_{c \bar{o}}+\boldsymbol{C}_{o b}{ }^{H} \boldsymbol{C}_{o b}, \\
& \overline{\boldsymbol{S}}_{c \bar{o}}=\boldsymbol{A}_{c \bar{o}}^{H}\left(\overline{\boldsymbol{S}}_{\bar{c}}-\overline{\boldsymbol{S}}_{\bar{c} \bar{b}} \boldsymbol{B}_{c \bar{o}}\left(l+\boldsymbol{B}_{c \bar{c}}^{H} \overline{\boldsymbol{S}}_{\bar{c} \bar{b}} \boldsymbol{B}_{c \bar{o}}\right)^{-1} \boldsymbol{B}_{c \bar{o}}^{H} \overline{\boldsymbol{S}}_{c \bar{o}}\right) \boldsymbol{A}_{c \bar{o}}+\boldsymbol{C}_{o b}{ }^{H} \boldsymbol{C}_{o b} .
\end{aligned}
$$

It then follows that $\boldsymbol{K}_{c \bar{o}}=\overline{\boldsymbol{K}}_{\bar{c} \overline{0}}$. 

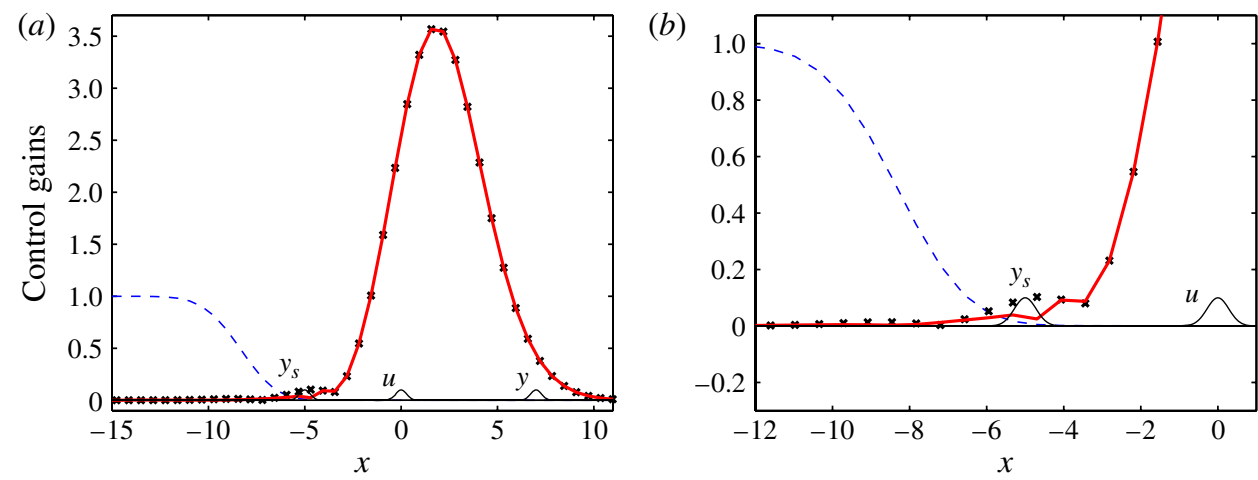

FIgURE 24. (Colour online) The control gains $\boldsymbol{K}$ and $\overline{\boldsymbol{K}}$ designed from $\boldsymbol{A}$ (full line; red online) and from $\boldsymbol{A}-\boldsymbol{L} \boldsymbol{C}_{s}$ (crosses), respectively, are compared, as a function of $x$. On the same plot the relative estimation error is presented as a dashed line. Panel $(b)$ is a zoom of panel $(a)$, focusing on the spy sensor location.

In our quest to compare the two control gains $\boldsymbol{K}$ and $\overline{\boldsymbol{K}}$, we still have to assess the equivalence of the observable and controllable parts, $\boldsymbol{K}_{c o}$ and $\overline{\boldsymbol{K}}_{c o}$. For the case of a convection-dominated flow, the observable part of the estimated state is mainly upstream of the spy sensor, while the controllable part of the estimated state (non-zero components of the estimated state) is downstream of it. The part of the state that is simultaneously controllable and observable, denoted by $X_{c o}$, is thus located in the vicinity of the upstream spy sensor.

From this situation, two cases arise. For convection-dominated flows, the controllable-observable region is rather small, and, in the limit of pure convection, $\boldsymbol{K}_{c o}$ and $\overline{\boldsymbol{K}}_{c o}$ have zero dimension. Then, the two control gains $\boldsymbol{K}$ and $\overline{\boldsymbol{K}}$ coincide. In a second case, consider the situation where the spy sensor is sufficiently upstream such that the estimation of the state in the region near $y_{s}$ has no influence on the control $u$ further downstream; consequently, $\boldsymbol{K}_{c o}$ as well as $\overline{\boldsymbol{K}}_{c o}$ are small or negligible. This is exemplified in figure 24 where the control gains $\boldsymbol{K}$ and $\overline{\boldsymbol{K}}$ are compared for the complex Ginzburg-Landau equation with the high convection speed $U_{r}=3$. Equivalence is observed throughout the computational domain, except for a small region near the spy sensor location. When placing $y_{s}$ even further upstream (not shown), the control gain in this small region drastically diminishes. For comparison, the relative estimation error is also included in the figures.

In conclusion, we observe that, for convection-dominated flows with a spy sensor placed sufficiently upstream, the two control strategies are equivalent and the feedforward control set-up inherits the optimality property of LQG control. This study explains the remarkable efficiency of the feed-forward controller observed previously in figure 15 and in the backward-facing step geometry.

\section{Discussion and conclusions}

In this paper, the control of convection-dominated amplifier flows has been investigated. It was observed that using a feedback control strategy becomes significantly less efficient when applied to convection-dominated situations. The reason is that, in such flows, information mainly travels downstream. Hence, sensors essentially describe the future flow state downstream but provide only limited 
knowledge of the flow upstream. Therefore any feedback information from a sensor located too far downstream is useless. To quantify this observation, the concept of visibility length was introduced in order to measure how far upstream a sensor is able to accurately estimate the flow. Not surprisingly, the visibility length was observed to decrease when the flow became more convection-dominated. These findings suggested the use of a feed-forward approach for the control of amplifier flows. Many approaches for the design of feed-forward controllers have been developed in the control literature, as noted in Qin \& Badgwell (2003). When applied to fluid flows, the procedure relies on the addition of spy sensors introduced upstream in order to measure the perturbations ahead of time. In this paper, the technique was based on subspace system identification followed by optimal controller design. System identification provided a model derived from sensor and actuator signals only. Such an approach is realistic since all these data are accessible in real experiments. In addition, when applied to a flow model based on the Ginzburg-Landau equation, the feed-forward control efficiency was found to be comparable to the optimal fullstate control. The technique was then validated on the two-dimensional flow over a backward-facing step at $R e=350$. The system was excited by three independent noise sources. Two spy sensors, one actuator and one objective sensor were used. The application of the present feed-forward control led to the reduction of the perturbation norms by a factor of 10 . The feed-forward method presented in this paper has the advantage of being amenable to direct numerical and theoretical comparison with the LQG control framework commonly used in fluid mechanics. It was observed on the Ginzburg-Landau model that the feed-forward control is almost as efficient as the optimal LQG control. The main advantage of the present technique, however, is that it only relies on experimentally accessible data. More generally, it was demonstrated that the two techniques are equivalent for sufficiently upstream spy sensor locations or for sufficiently convective flows. Under these conditions the feed-forward approach was proved to be optimal.

In contrast to the LQG and linear-quadratic regulator (LQR) formulations, feedforward techniques have rarely been used for the control of flow instabilities, with the exception of the numerical study of Hervé et al. (2012) and the experimental investigation of Rathnasingham \& Breuer (2003). Several reasons may explain this observation. In the plane Poiseuille flow amplifier, the translational invariance in the streamwise direction is often exploited to impose streamwise periodicity (Joshi, Speyper \& Kim 1997). In such a case, perturbations at the outlet are re-introduced at the inlet and the distinction between upstream and downstream becomes elusive. Furthermore, in most theoretical studies, sensors (shear stress gauges) and actuators (blowing or suction devices) are assumed to be continuously distributed along the wall, whereas in real flows only a discrete set of localized sensors and actuators is feasible. In all these instances, the relative positions of sensors and actuators are difficult to define. In order to remedy this lack of realism, the present work could be extended to the application of the feed-forward identification and feed-forward control to plane Poiseuille flow. It should be noted that feed-forward control has already been applied successfully numerically on a flat-plate boundary layer (Bagheri et al. 2009a; Semeraro et al. 2011). Although the method was referred to as feedback control by the authors, the approach was essentially feed-forward since upstream spy sensors were used and the feedback information provided by the downstream objective sensors was clearly negligible in both studies. More generally, the feedback control terminology is often used in fluid mechanics to designate an optimal LQR or LQG control, independently of the relative positions of sensors and actuators. According to 
the theoretical study presented in $\S 6.2$ and the numerical evidence given in $\S 4.2$, one may expect the realizable feed-forward technique of the present study to be equivalent to and as efficient as the traditional LQG control.

The control of the backward-facing step performed by Barbagallo et al. (2012) may, at first sight, be considered as a counter-example to the above conclusions: based only on the knowledge of a downstream sensor, the controller was able to achieve a sufficiently accurate state estimation in order to efficiently control the flow. However, among the four sensor positions considered in Barbagallo et al. (2012), the best results were obtained for the most upstream sensor placement. This finding confirms our intuition, based on the concept of visibility length, that a sensor provides accurate upstream estimation only in its own immediate neighbourhood.

The successful validation of the feed-forward approach in amplifier flows gives us confidence that an experimental implementation is feasible. In order to do so, a few conceptual and experimental issues will have to be addressed. In particular, the analysis relies on the linearity of the fluid flow, and this property may not be preserved when large transient growth (Blackburn et al. 2008) may be expected. Linear control in flows governed by the nonlinear Navier-Stokes equations has, however, been the subject of many numerical studies, and adequate robustness has usually been obtained (Högberg, Bewley \& Henningson 2003; Kim \& Bewley 2007; Sharma et al. 2011; Hervé et al. 2012). In addition, although sensors may be faithfully represented in numerical simulations, modelling actuators by simple localized volume forces is a convenient but strongly idealized representation. In practice, most realistic actuators are directly mounted on the walls and behave nonlinearly. Finally, a strong limiting feature of the present analysis is its two-dimensional nature. The previously cited work of Semeraro et al. (2011) gives us confidence that the application of the technique to three-dimensional observations is possible. The experimental implementation of the feed-forward control procedure is currently under way for the backward-facing step configuration.

\section{Acknowledgement}

The support of Ecole Polytechnique and of the Partner University Fund (PUF) is gratefully acknowledged.

\section{Appendix A. Subspace identification algorithm}

In its general form, a linear time-invariant (LTI) system with $m$ inputs and $l$ outputs may be written in state-space form as

$$
\begin{aligned}
q_{k+1} & =\boldsymbol{A} q_{k}+\boldsymbol{B} u_{k}+\boldsymbol{B}_{w} w_{k}, \\
y_{k} & =\boldsymbol{C} q_{k}+\boldsymbol{D} u_{k}+v_{k} .
\end{aligned}
$$

If the system order is $n$, then the state matrix $\boldsymbol{A}$ is $n \times n$, the input matrix $\boldsymbol{B}$ is $n \times m$, the output matrix $\boldsymbol{C}$ is $l \times n$ and the direct matrix $\boldsymbol{D}$ is $l \times m$. In practice, the direct matrix $\boldsymbol{D}$ is often either negligible or equal to zero. In addition, the noise covariances $\boldsymbol{Q}, \boldsymbol{R}$ and $\boldsymbol{S}$ are defined as in $\S 2$. The purpose of the subspace identification algorithm is to recover the system matrices $\boldsymbol{A}, \boldsymbol{B}, \boldsymbol{C}, \boldsymbol{D}$ and the noise covariances $\boldsymbol{Q}, \boldsymbol{R}, \boldsymbol{S}$ from the knowledge of input-output data sequences only. In order to describe the technique, preliminary mathematical manipulations of the system (A 1) have to be performed. 
A.1. Reformulation of the discrete state-space system

It is convenient to rearrange the discrete state-space system (A 1) into several equivalent formulations that emphasize either prediction or estimation (Qin 2006).

A central concept in subspace identification is the innovation form of the original system (A 1) defined by

$$
\begin{aligned}
q_{k+1} & =\boldsymbol{A} q_{k}+\boldsymbol{B} u_{k}+\boldsymbol{L} e_{k}, \\
y_{k} & =\boldsymbol{C} q_{k}+\boldsymbol{D} u_{k}+e_{k},
\end{aligned}
$$

where $\boldsymbol{L}$ is the steady Kalman gain obtained from the solution of a discrete Riccati equation and $e_{k}$ is a Gaussian white noise with second-order moment $\mathscr{E}\left(e_{k} e_{p}^{H}\right)=\boldsymbol{R} \delta_{p k}$. This formulation, which is based on the same Kalman gain as in the familiar estimation problem $(\$ 3.2)$, is equivalent to (A 1) in the sense that the deterministic parts of the outputs are identical while the stochastic parts of the output show the same statistical moments.

Alternatively, one may easily recast the state-space system (A 2) into the predictor form (Qin 2006)

$$
\begin{aligned}
q_{k+1} & =\boldsymbol{A}_{L} q_{k}+\boldsymbol{B}_{L} z_{k}, \\
y_{k} & =\boldsymbol{C} q_{k}+\boldsymbol{D} u_{k}+e_{k},
\end{aligned}
$$

where $z_{k}=\left(u_{k} y_{k}\right)^{\mathrm{T}}, \boldsymbol{A}_{L}=\boldsymbol{A}-\boldsymbol{L} \boldsymbol{C}$ and $\boldsymbol{B}_{L}$ is the block matrix $(\boldsymbol{B}-\boldsymbol{L} \boldsymbol{D}, \boldsymbol{L})$. The advantage of this formulation is that the (unknown) noise $e_{k}$ does not appear explicitly in the state equation (A $3 a$ ) but is accounted for in the known (noise-contaminated) measurement $y_{k}$. It should be stressed again that systems (A 1)-(A 3) are all equivalent in the above-defined sense. They will be used interchangeably in the analysis that follows.

In the next step, the above one-step vector-based linear difference equations are reformulated into multi-step matrix-based expressions. Two extended states $\boldsymbol{X}_{p}=$ $\left(q_{k}, q_{k+1}, \ldots, q_{k+j-1}\right)$ and $\boldsymbol{X}_{f}=\left(q_{k+i}, q_{k+i+1}, \ldots, q_{k+i+j-1}\right)$ are introduced by stacking vector states $q_{k}$. The subscript $p$ in $\boldsymbol{X}_{p}$ stands for past states; the subscript $f$ in $\boldsymbol{X}_{f}$ denotes future states. Both state sequences, $\boldsymbol{X}_{f}$ and $\boldsymbol{X}_{p}$, contain $j$ columns describing the state at $j$ consecutive time steps and are shifted by $i$ time steps, where $i \ll j$ (figure 25). In addition, the shift $i$ is chosen to be larger than the system order $n$. Starting with the predictor form (A $3 a$ ) of the dynamical system, the matrix form of the state equation is sought by recursive iteration, which results in

$$
\boldsymbol{X}_{f}=\boldsymbol{A}_{L}^{i} \boldsymbol{X}_{p}+\boldsymbol{\Delta}_{c} \boldsymbol{Z}_{p}
$$

where

$$
\boldsymbol{\Delta}_{c}=\left(\begin{array}{llll}
\boldsymbol{B}_{L} & \boldsymbol{A}_{L} \boldsymbol{B}_{L} & \ldots & \boldsymbol{A}_{L}^{i-1} \boldsymbol{B}_{L}
\end{array}\right), \quad \boldsymbol{z}_{p}=\left(\begin{array}{cccc}
z_{k} & z_{k+1} & \ldots & z_{k+j-1} \\
z_{k+1} & z_{k+2} & \ldots & z_{k+j} \\
\vdots & \vdots & \ddots & \vdots \\
z_{k+i-1} & z_{k+i} & \ldots & z_{k+j+i-2}
\end{array}\right),
$$

and $\boldsymbol{A}_{L}^{i}$ represents $\boldsymbol{A}_{L}$ applied $i$ times. In addition, a similar recursive iteration technique, this time applied to the innovation form (A 2), yields the matrix form of the measurement equation,

$$
\boldsymbol{Y}_{f}=\boldsymbol{\Gamma}_{i} \boldsymbol{X}_{f}+\boldsymbol{H}_{i} \boldsymbol{U}_{f}+\boldsymbol{G}_{i} \boldsymbol{E}_{f}
$$


System equations in VECTOR form

$$
\begin{gathered}
\text { One time step } \\
\left\{\begin{array}{l}
q_{k} \\
q_{k+1}=\boldsymbol{A}_{L} q_{k}+\boldsymbol{B}_{L} z_{k} \\
y_{k+1}=\boldsymbol{C}_{q_{k+1}}+\boldsymbol{D} u_{k+1}+e_{k+1}
\end{array}\right.
\end{gathered}
$$

System equations in MATRIX form

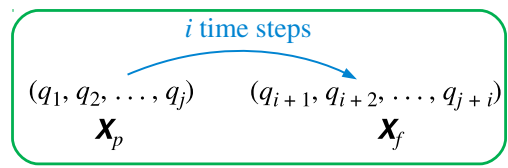

$\left\{\begin{array}{l}\boldsymbol{x}_{f}=\boldsymbol{A}_{L}^{i} \boldsymbol{X}_{p}+\boldsymbol{\Delta}_{c} \boldsymbol{Z}_{p} \\ \boldsymbol{Y}_{f}=\boldsymbol{\Gamma}_{i} \boldsymbol{x}_{f}+\boldsymbol{H}_{i} \boldsymbol{U}_{f}+\boldsymbol{G}_{i} \boldsymbol{E}_{f}\end{array}\right.$

FIGURE 25. (Colour online) Comparison of the system equations in vector form (A 3) and in matrix form (A 4) and (A 6).

with

$$
\begin{aligned}
& \Gamma_{i}=\left(\begin{array}{c}
C \\
C A \\
\vdots \\
C A^{i-1}
\end{array}\right) \\
& \boldsymbol{H}_{i}=\left(\begin{array}{ccccc}
\boldsymbol{D} & 0 & 0 & \ldots & 0 \\
\boldsymbol{C B} & \boldsymbol{D} & 0 & \ldots & 0 \\
\boldsymbol{C A B} & \boldsymbol{C B} & \boldsymbol{D} & \ldots & 0 \\
\vdots & \vdots & \vdots & \ddots & \vdots \\
\boldsymbol{C A} \boldsymbol{A}^{i-2} \boldsymbol{B} & \boldsymbol{C A}^{i-3} \boldsymbol{B} & \boldsymbol{C A}^{i-4} \boldsymbol{B} & \ldots & \boldsymbol{D}
\end{array}\right), \\
& \boldsymbol{G}_{i}=\left(\begin{array}{ccccc}
\boldsymbol{I} & 0 & 0 & \ldots & 0 \\
\boldsymbol{C L} & \boldsymbol{I} & 0 & \ldots & 0 \\
\boldsymbol{C A L} & \boldsymbol{C L} & \boldsymbol{I} & \ldots & 0 \\
\vdots & \vdots & \vdots & \ddots & \vdots \\
C A^{i-2} \boldsymbol{L} & \boldsymbol{C A}^{i-3} \boldsymbol{L} & \boldsymbol{C A}^{i-4} \boldsymbol{L} & \ldots & \boldsymbol{I}
\end{array}\right) \\
& \boldsymbol{Y}_{f}=\left(\begin{array}{cccc}
y_{k+i} & y_{k+i+1} & \ldots & y_{k+i+j-1} \\
y_{k+i+1} & y_{k+i+2} & \ldots & y_{k+i+j} \\
\vdots & \vdots & \ddots & \vdots \\
y_{k+2 i-1} & y_{k+2 i} & \ldots & y_{k+j+2 i-2}
\end{array}\right) \text {. }
\end{aligned}
$$

The quantities $\boldsymbol{U}_{f}$ and $\boldsymbol{E}_{f}$ are identical in structure to $\boldsymbol{Y}_{f}$ : they are characterized by a Hankel matrix pattern where $y$ is changed into $u$ or $e$, respectively. Combining the state equation (A 4) and the measurement equation (A 6) in their matrix form yields the final equation

$$
\boldsymbol{Y}_{f}=\boldsymbol{\Gamma}_{i} \boldsymbol{\Delta}_{c} \boldsymbol{Z}_{p}+\boldsymbol{\Gamma}_{i} \boldsymbol{A}_{L}^{i} \boldsymbol{X}_{p}+\boldsymbol{H}_{i} \boldsymbol{U}_{f}+\boldsymbol{G}_{i} \boldsymbol{E}_{f}
$$

which plays a central role in system identification by subspace techniques (Qin 2006). 
A.2. Extraction of the system matrices by subspace projections

Before proceeding to the solution algorithm for (A 8), the following assumptions are introduced, which will prove advantageous for the procedural extraction of the system matrices and noise covariances. The matrix $\boldsymbol{A}_{L}$, which describes the estimator dynamics, is taken as strictly stable, with all eigenvalues confined inside the unit disk, under mild assumptions. Furthermore, the input $u_{k}$ is uncorrelated to the noise $e_{k}$, which is a consequence of the open-loop nature of our identification method. Moreover, the input $u_{k}$ is taken as sufficiently rich in temporal behaviour to excite all the observable dynamics of the system. These reasonable assumptions will be brought to bear step-by-step in the further development of the identification algorithm.

\section{A.2.1. Extraction of the extended observability matrix $\Gamma_{i}$}

For convenience, we take the limit $i \rightarrow \infty$ and, recalling the contractive property of the matrix $\boldsymbol{A}_{L}$, we obtain $\boldsymbol{A}_{L}^{i} \rightarrow 0$. However, the final results obtained below may be shown to be valid even for finite $i$ (Van Overschee \& De Moor 1994, 1996). In the asymptotic limit, (A 4) and (A 8) simplify to

$$
\begin{aligned}
\boldsymbol{X}_{f} & =\boldsymbol{\Delta}_{c} \boldsymbol{Z}_{p}, \\
\boldsymbol{Y}_{f} & =\left(\begin{array}{ll}
\boldsymbol{\Gamma}_{i} \boldsymbol{\Delta}_{c} & \boldsymbol{H}_{i}
\end{array}\right)\left(\begin{array}{l}
\boldsymbol{Z}_{p} \\
\boldsymbol{U}_{f}
\end{array}\right)+\boldsymbol{G}_{i} \boldsymbol{E}_{f} .
\end{aligned}
$$

In the above equations, the quantities $\boldsymbol{Z}_{p}$ and $\boldsymbol{U}_{f}$, which involve $u$ and $y$ only, are known since they are either imposed or measured. The remaining variables, except $\boldsymbol{E}_{f}$, implicitly contain the unknown system matrices.

The purpose of the next few steps is to recover the matrices $\boldsymbol{\Gamma}_{i} \boldsymbol{\Delta}_{c}$, and then $\boldsymbol{\Gamma}_{i}$. We proceed by right-multiplying equation (A $\left.9 b\right)$ by $\left(\boldsymbol{Z}_{p}^{\mathrm{T}} \boldsymbol{U}_{f}^{\mathrm{T}}\right)$. We then use the previous assumptions that past measurements $\boldsymbol{Z}_{p}$ as well as future control $\boldsymbol{U}_{f}$ are uncorrelated with the white noise $\boldsymbol{E}_{f}$. This manipulation eliminates the term $\boldsymbol{G}_{i} \boldsymbol{E}_{f}$ from the above equation. In the remaining terms, the expression $\left(\begin{array}{l}\boldsymbol{z}_{p} \\ \boldsymbol{U}_{f}\end{array}\right)\left(\begin{array}{ll}\boldsymbol{Z}_{p}^{\mathrm{T}} & \boldsymbol{U}_{f}^{\mathrm{T}}\end{array}\right)$ is known, as is $\boldsymbol{Y}_{f}\left(\boldsymbol{Z}_{p}^{\mathrm{T}} \boldsymbol{U}_{f}^{\mathrm{T}}\right)$, from which the unknowns $\boldsymbol{\Gamma}_{i} \boldsymbol{\Delta}_{c}$ and $\boldsymbol{H}_{i}$ can now be determined by a straightforward least-squares technique followed by matrix partitioning in order to isolate $\boldsymbol{\Gamma}_{i} \boldsymbol{\Delta}_{c}$.

At this point, we have determined the quantity $\boldsymbol{\Gamma}_{i} \boldsymbol{\Delta}_{c} \boldsymbol{Z}_{p}$, which, using (A $9 a$ ), is equivalent to $\boldsymbol{\Gamma}_{i} \boldsymbol{X}_{f}$. The splitting of this matrix product relies on the last of our assumptions (excitation of all dynamical states), which ensures a full row rank of $\boldsymbol{X}_{f}$. In addition, $\boldsymbol{\Gamma}_{i}$ also has full column rank under the assumption of full observability. These properties suggest an application of a singular value decomposition: (i) to determine the order of the identified system as the rank of $\boldsymbol{\Gamma}_{i} \boldsymbol{X}_{f}$; and (ii) to isolate $\boldsymbol{\Gamma}_{i}$ from the product $\boldsymbol{\Gamma}_{i} \boldsymbol{X}_{f}$. Mathematically, this amounts to

$$
\boldsymbol{\Gamma}_{i} \boldsymbol{X}_{f}=\left(\begin{array}{ll}
\boldsymbol{U}_{1} & \boldsymbol{U}_{2}
\end{array}\right)\left(\begin{array}{cc}
\boldsymbol{\Sigma}_{1} & 0 \\
0 & \boldsymbol{\Sigma}_{2}
\end{array}\right)\left(\begin{array}{l}
\boldsymbol{V}_{1}^{H} \\
\boldsymbol{V}_{2}^{H}
\end{array}\right),
$$

where the diagonal matrix $\boldsymbol{\Sigma}$ has been partitioned so that $\left\|\boldsymbol{\Sigma}_{2}\right\|$ is negligible compared to $\left\|\boldsymbol{\Sigma}_{1}\right\|$. The size of $\boldsymbol{\Sigma}_{1}$ then represents the order of the identified system. In addition we obtain the extended observability matrix $\boldsymbol{\Gamma}_{i}$ as

$$
\Gamma_{i}=\boldsymbol{U}_{1} \Sigma_{1}^{1 / 2} .
$$


Even though this result has been derived under the assumption $i \rightarrow \infty$, it can be shown (Van Overschee \& De Moor 1996) that $\boldsymbol{\Gamma}_{i}$ is still equal to $\boldsymbol{U}_{1} \boldsymbol{\Sigma}_{1}^{1 / 2}$ when $i$ is finite.

Based on the knowledge of $\boldsymbol{\Gamma}_{i}$, two different approaches may be adopted. If the noise covariances are not needed, then a simple algorithm can be used for the computation of the system matrices $\boldsymbol{A}, \boldsymbol{B}, \boldsymbol{C}, \boldsymbol{D}$ only. This approach is referred to as 'simulation focus', because the identified model is particularly accurate for the simulation of unknown outputs from known inputs, without taking into account the noise sources. In particular, this approach is relevant for the feed-forward identification presented in $\S 4.1$. However, if the noise covariances are sought, then a second technique consists in identifying the system in its innovation form. This approach is referred to as 'estimation focus', because it directly provides an approximation of the Kalman gain used in optimal estimation processes. Both procedures are outlined below.

\section{A.2.2. A first technique: 'simulation focus'}

The first step consists in deriving the system matrices $\boldsymbol{A}$ and $\boldsymbol{C}$ from $\boldsymbol{\Gamma}_{i}$. To this end, we repeat the above procedure to obtain $\boldsymbol{\Gamma}_{i-1}$, which, by definition, is related to $\boldsymbol{\Gamma}_{i}$ via

$$
\left(\begin{array}{cc}
I & 0 \\
0 & \Gamma_{i-1}
\end{array}\right)\left(\begin{array}{l}
\boldsymbol{C} \\
\boldsymbol{A}
\end{array}\right)=\Gamma_{i} .
$$

This system can easily be solved for $\boldsymbol{A}$ and $\boldsymbol{C}$ by least-squares techniques. To determine the remaining matrices $\boldsymbol{B}$ and $\boldsymbol{D}$, one observes that the problem is linear in these matrices, and a matching to the output data may be used to find them (McKelvey 1994; Van Overschee \& De Moor 1996).

\section{A.2.3. A second technique: 'estimation focus'}

The general idea behind the technique presented below is to identify the matrices $\boldsymbol{A}$, $\boldsymbol{B}, \boldsymbol{C}, \boldsymbol{D}$ based on the system written in its innovation form. For this, two consecutive estimations of the extended state are obtained. Then, the system matrices are computed by least-squares technique and the residual provides an approximation of the noise covariances.

In the previous derivation of $\boldsymbol{\Gamma}_{i}$, we took advantage of the limit $i \rightarrow \infty$, which corresponds to an infinite shift between the past and future extended states, and we eliminated the dependence on the matrix $\boldsymbol{A}_{L}$. Consequently, we could express the future extended state $\boldsymbol{X}_{f}$ as a function of the (known) past data $\boldsymbol{Z}_{p}$ and the (known) future input $\boldsymbol{U}_{f}$; see $(\mathrm{A} 9 a)$. This relation no longer holds for a finite shift $i$, and the best we can do, using the available data, is to replace the true state by an optimal (Kalman) estimate (see Van Overschee \& De Moor $(1994,1995)$ for a rigorous proof). Following the same least-squares technique that has been applied to (A9), we may then compute the intermediate quantity defined as

$$
\boldsymbol{R}_{i} \equiv \boldsymbol{\Gamma}_{i} \hat{\boldsymbol{X}}_{f, i}+\boldsymbol{H}_{i} \boldsymbol{U}_{f}
$$

where $\hat{\boldsymbol{X}}_{f, i}$ denotes an $i$-step Kalman estimate of the future extended state $\boldsymbol{X}_{f}$ starting at index $i$. Repeating the same computation for a shifted index $i+1$, we obtain

$$
\boldsymbol{R}_{i+1} \equiv \boldsymbol{\Gamma}_{i-1} \hat{\boldsymbol{x}}_{f, i+1}+\boldsymbol{H}_{i} \boldsymbol{U}_{f}^{-},
$$

where $\boldsymbol{U}_{f}^{-}$is defined from $\boldsymbol{U}_{f}$ by removing the first $m$ rows, $m$ being the number of inputs. 
Since $\hat{\boldsymbol{X}}_{f, i}$ and $\hat{\boldsymbol{X}}_{f, i+1}$ are consecutive estimates of the same unsteady Kalman filter, they are governed by

$$
\left(\begin{array}{c}
\hat{\boldsymbol{X}}_{f, i+1} \\
\boldsymbol{Y}_{i}
\end{array}\right)=\left(\begin{array}{cc}
\boldsymbol{A} & \boldsymbol{B} \\
\boldsymbol{C} & \boldsymbol{D}
\end{array}\right)\left(\begin{array}{c}
\hat{\boldsymbol{X}}_{f, i} \\
\boldsymbol{U}_{i}
\end{array}\right)+\left(\begin{array}{c}
\boldsymbol{L}_{i} \boldsymbol{E}_{i} \\
\boldsymbol{E}_{i}
\end{array}\right)
$$

where $\boldsymbol{Y}_{i}=\left(\begin{array}{llll}y_{k+i} & y_{k+i+1} & \ldots & y_{k+i+j-1}\end{array}\right)$ and $\boldsymbol{U}_{i}=\left(\begin{array}{llll}u_{k+i} & u_{k+i+1} & \ldots & u_{k+i+j-1}\end{array}\right)$. By definition of the Kalman filter, the quantity $\boldsymbol{E}_{i}$ is orthogonal to $\boldsymbol{U}_{f}$ and $\hat{\boldsymbol{X}}_{f, i}$. If the estimated extended states $\hat{\boldsymbol{X}}_{f, i}$ and $\hat{\boldsymbol{X}}_{f, i+1}$ were known, we could use this orthogonality in (A 15) to solve for $(\boldsymbol{A}, \boldsymbol{B}, \boldsymbol{C}, \boldsymbol{D})$ by least-squares techniques. However, because $\hat{\boldsymbol{X}}_{f, i}$ depends on $\boldsymbol{H}_{i}$ (see (A 13)), which in turn depends on the system matrices, we have to use in (A 15) the expressions deduced from (A 13) and (A 14),

$$
\begin{aligned}
\hat{\boldsymbol{X}}_{i} & =\boldsymbol{\Gamma}_{i}^{\dagger}\left(\boldsymbol{R}_{i}-\boldsymbol{H}_{i} \boldsymbol{U}_{f}\right), \\
\hat{\boldsymbol{X}}_{i+1} & =\boldsymbol{\Gamma}_{i-1}^{\dagger}\left(\boldsymbol{R}_{i+1}-\boldsymbol{H}_{i} \boldsymbol{U}_{f}^{-}\right),
\end{aligned}
$$

in order to extract the unknown system matrices $(\boldsymbol{A}, \boldsymbol{B}, \boldsymbol{C}, \boldsymbol{D})$. In the above expressions, the pseudo-inverse $\boldsymbol{\Gamma}_{i}^{\dagger}$ satisfies $\boldsymbol{\Gamma}_{i}^{\dagger} \boldsymbol{\Gamma}_{i}=\boldsymbol{I}$ and it exists since $\boldsymbol{\Gamma}_{i}$ has full column rank. Substituting (A 16) and (A 17) into (A 15) and rearranging the terms yields

$$
\left(\begin{array}{c}
\boldsymbol{\Gamma}_{i-1}^{\dagger} \boldsymbol{R}_{i+1} \\
\boldsymbol{Y}_{i}
\end{array}\right)=\left(\begin{array}{cc}
\boldsymbol{A} & \boldsymbol{K}_{1} \\
\boldsymbol{C} & \boldsymbol{K}_{2}
\end{array}\right)\left(\begin{array}{c}
\boldsymbol{\Gamma}_{i}^{\dagger} \boldsymbol{R}_{i} \\
\boldsymbol{U}_{f}
\end{array}\right)+\left(\begin{array}{c}
\boldsymbol{L}_{i} \boldsymbol{E}_{i} \\
E_{i}
\end{array}\right)
$$

with

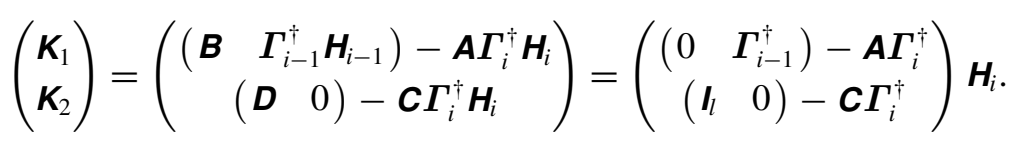

We can now easily determine the matrix $\left(\begin{array}{ll}A & \boldsymbol{K}_{1} \\ \boldsymbol{C} & \boldsymbol{K}_{2}\end{array}\right)$ in (A 18) by least-squares methods. As before, the system matrices $\boldsymbol{A}$ and $\boldsymbol{C}$ follow directly from the least-squares solution by appropriate matrix partitioning. The matrices $\boldsymbol{B}$ and $\boldsymbol{D}$ are implicitly, but linearly, contained in $\boldsymbol{K}_{1}$ and $\boldsymbol{K}_{2}$ and can be factored out and solved for. The algebraic manipulations are rather cumbersome and omitted here; for details see Van Overschee \& De Moor (1996).

In a final step, the noise covariances $\boldsymbol{R}, \boldsymbol{Q}$ and $\boldsymbol{S}$ are determined by processing the residual of (A 18) with all system matrices $\boldsymbol{A}, \boldsymbol{B}, \boldsymbol{C}, \boldsymbol{D}$ determined. We have

$$
\left(\begin{array}{c}
\boldsymbol{L}_{i} \boldsymbol{E}_{i} \\
\boldsymbol{E}_{i}
\end{array}\right)=\left(\begin{array}{c}
\boldsymbol{\Gamma}_{i-1}^{\dagger} \boldsymbol{R}_{i+1} \\
\boldsymbol{Y}_{i}
\end{array}\right)-\left(\begin{array}{cc}
\boldsymbol{A} & \boldsymbol{K}_{1} \\
\boldsymbol{C} & \boldsymbol{K}_{2}
\end{array}\right)\left(\begin{array}{c}
\boldsymbol{\Gamma}_{i}^{\dagger} \boldsymbol{R}_{i} \\
\boldsymbol{U}_{f}
\end{array}\right),
$$

from which the noise covariances follow according to

$$
\left(\begin{array}{cc}
\boldsymbol{R} & \boldsymbol{S} \\
\boldsymbol{S}^{H} & \boldsymbol{Q}
\end{array}\right)=\left(\begin{array}{c}
\boldsymbol{L}_{i} E_{i} \\
\boldsymbol{E}_{i}
\end{array}\right)\left(\begin{array}{c}
\boldsymbol{L}_{i} E_{i} \\
\boldsymbol{E}_{i}
\end{array}\right)^{\mathrm{T}}
$$

This step concludes the identification of the system matrices and noise covariances from input and output sequences. This procedure eliminates the need to rely on specific system matrices. As a component in the full control design, it ensures a realistic approach that is also applicable in experiments. 
Note that the general subspace identification procedure may be further modified by introducing weight matrices $\boldsymbol{W}_{1,2}$ into the left-hand side of (A 10) in the form $\boldsymbol{W}_{1} \boldsymbol{\Gamma}_{i} \boldsymbol{X}_{f} \boldsymbol{W}_{2}$ and by performing the singular value decomposition on the latter expression. Several special choices of $\boldsymbol{W}_{1}$ and $\boldsymbol{W}_{2}$ correspond to well-known algorithms, such as CVA (Larimore 1983, 1990), MOESP (Verhaegen \& Deprettere 1991) and N4SID (Van Overschee \& De Moor 1994), and more details on the derivation of these schemes may be found in Van Overschee \& De Moor (1995). In this paper, the implementation of the MOESP weighting has been chosen; but it was verified that all three algorithms give comparable results.

\section{Appendix B. Choice of user parameters for subspace identification}

Before applying any system identification technique, several parameters have to be chosen. In particular, the number of samples, the type of input signal, the order of the model and the shift parameter $i$ are of crucial importance in subspace identification techniques. These user-specified parameters are studied in this appendix using the same configuration as in figure 16, but the convection speed is kept constant at $U_{r}=3$ and the above identification parameters are successively varied while their effect on control efficiency is monitored. It is useful to define the characteristic time of the system $\tau$ as the time for a wave packet to travel from the most upstream input (spy sensor at $x_{s}=-7$ ) to the most downstream output (objective sensor $x_{y}=7$ ). More precisely, the group velocity is $U_{r}+c_{d} U_{i}$ (Bagheri et al. 2009b), and thus we obtain

$$
\tau \equiv \frac{x_{y}-x_{s}}{U_{r}+c_{d} U_{i}} \approx 5.4
$$

for our configuration.

\section{B.1. Number of samples}

The quality of the input and output signals used for the identification has a direct impact on the quality of the resulting model. In particular, any system identification algorithm should give better results as the length of the input and output signals increases. This is verified in figure 26, where the efficiency of the feed-forward controller is represented as a function of the number of samples used in the identification step. It is observed that the efficiency of the control (full line with error bars; blue online) approaches the optimal LQG upper bound (full line with plus symbols; red online) as the number of samples increases. In this study, the order of the model and the shift parameter are kept constant and equal to 6 and 67, respectively. The input signal $u$ is white noise. Throughout this paper, the number of samples is chosen as 5000 (i.e. 500 time units). This value is represented by a vertical dotted line in figure 26. In practice, the length of the signals used for identification has to be significantly larger than any characteristic time scale of the system. The value used in this paper is nearly 100 times the characteristic time scale $\tau$.

\section{B.2. Identified model order}

In this section the choice of model order is analysed. In figure 27(a), the efficiency of the feed-forward controller is shown as a function of the model order. It appears that the control (and, consequently, the identification) is optimal for a model order between six and eight. Surprisingly, the performance of models decreases beyond this point. This can be explained by the fact that all accessible dynamics of the system has already been captured by eight modes, and that the remaining dynamics is not 


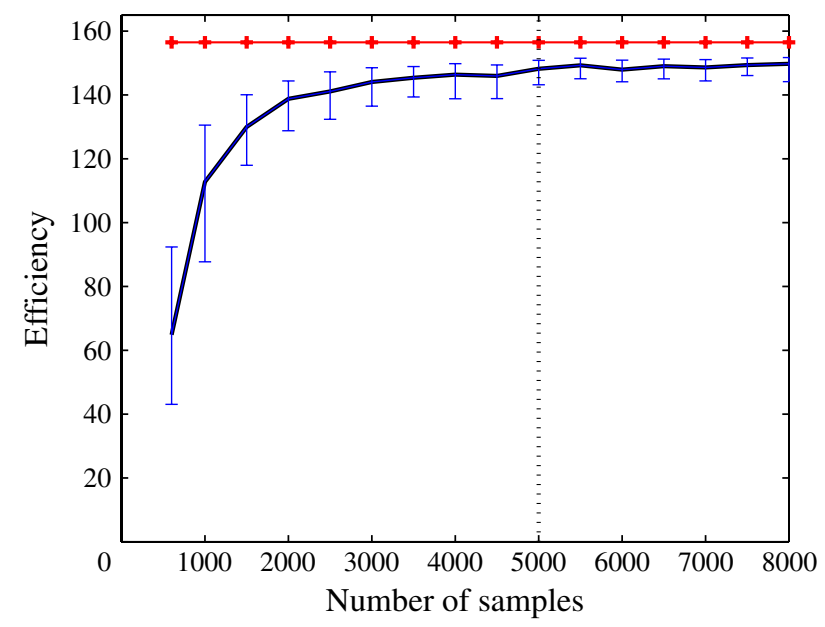

FIGURE 26. (Colour online) Control efficiency of the feed-forward approach as a function of the number of samples for the complex Ginzburg-Landau model. Fifty realizations of identification and control were performed; the average (full line with error bars; blue online) and the standard deviation are displayed. These curves are compared to the optimal LQG control designed from the full-order system (full line with plus symbols; red online).
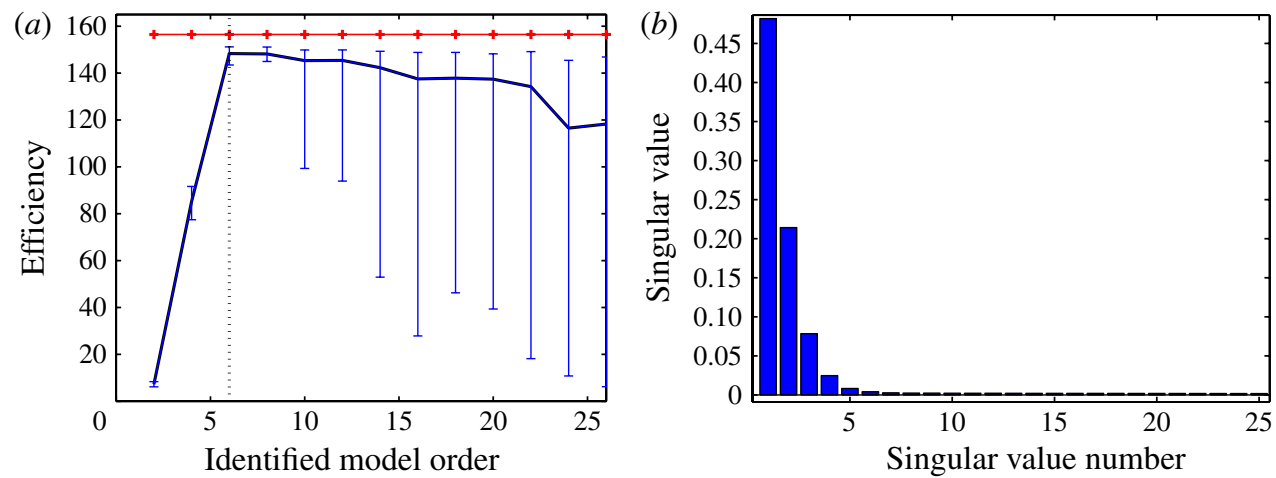

FIGURE 27. (Colour online) (a) Control efficiency of the feed-forward approach as a function of the identified model order for the complex Ginzburg-Landau model. Fifty realizations of identification and control were performed; the average (full line with error bars; blue online) and one standard deviation are plotted. These curves are compared to the optimal LQG control designed from the full-order system (full line with plus symbols; red online). (b) Singular values from (A 10) of the subspace identification process.

accessible and is essentially hidden within the measurement noise. Trying to extract additional information by increasing the model order only degrades the quality of the model. This is illustrated in figure $27(a)$ by the increasing standard deviation observed for higher-order models. In practice, however, the present parameter study may not be available a priori. Similar to many other system identification techniques, subspace identification relies on a singular value decomposition for the truncation of the order. This corresponds to (A 10) in the description of the technique. In figure $27(b)$ the singular values are represented in decreasing order. A sensible procedure is to select 


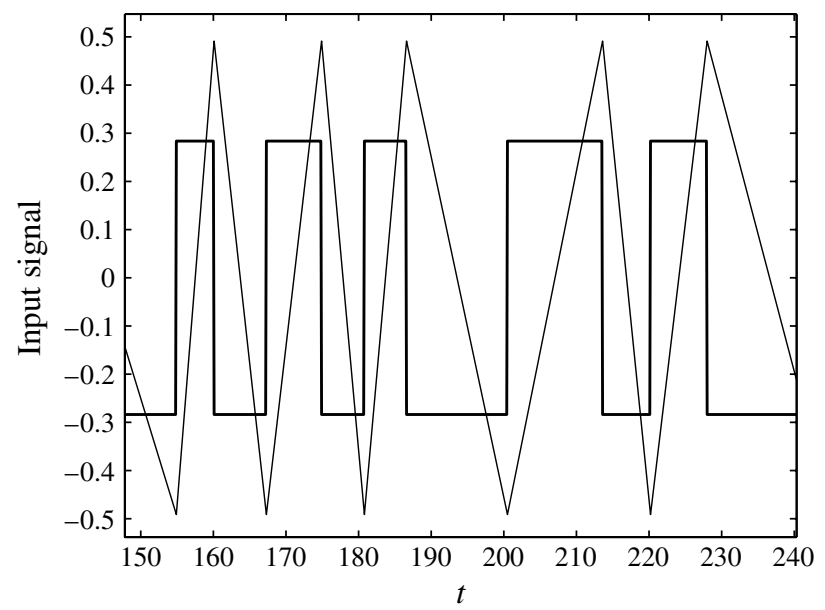

FIGURE 28. Samples of the random binary signal and of the random triangular signal. Both signals have the same standard deviation.

the order as the number of singular values above a given threshold. In this paper, better results were obtained by detecting a sudden drop in singular values; for our configuration, this technique gives an order of six. Except when specified otherwise, an automatic order detection is performed in this paper.

\section{B.3. Type of input signal}

In this paper, a white-noise signal is used as an input at the system identification stage. This signal has the advantage of exciting all frequencies of the system; however, in practice, real actuators are not capable of accomplishing such an excitation. Furthermore, it might be advantageous to tune the frequency content of the input signal to better force the dynamics of interest in the system. To this end, a random binary signal is often used as input signal. It is constructed by low-pass filtering a white-noise signal and by applying the sign function to the result; in this way, a random binary signal is generated. Its frequency content can be tailored to better excite the dynamics of the system. Similarly, a random triangular signal can be constructed by replacing the squares of the random binary signal by triangles. A representative sample of these two signals is shown in figure 28 , and the corresponding control efficiency is given in table 1 . We conclude that the random binary input signal produces a better control efficiency. In Ljung (1999) criteria for the choice of the input are given. In particular, it is argued that a proper input signal should have a standard deviation as close as possible to its maximum. This may be the reason why the random binary signal gives better performances than the other input signals. Other possible choices comprise, among others, chirp signals, sum of sinusoids or pseudo-random binary signals.

\section{B.4. Shift parameter}

In subspace identification the shift between the past extended state and the future extended state is a user-specified parameter. During the first projection step of the subspace algorithm, the future objective sensor measurements are described as a linear combination of the past spy and actuator signals. Hence, to be able to find any correlation between these quantities, it is imperative that the shift parameter $i$ is larger 


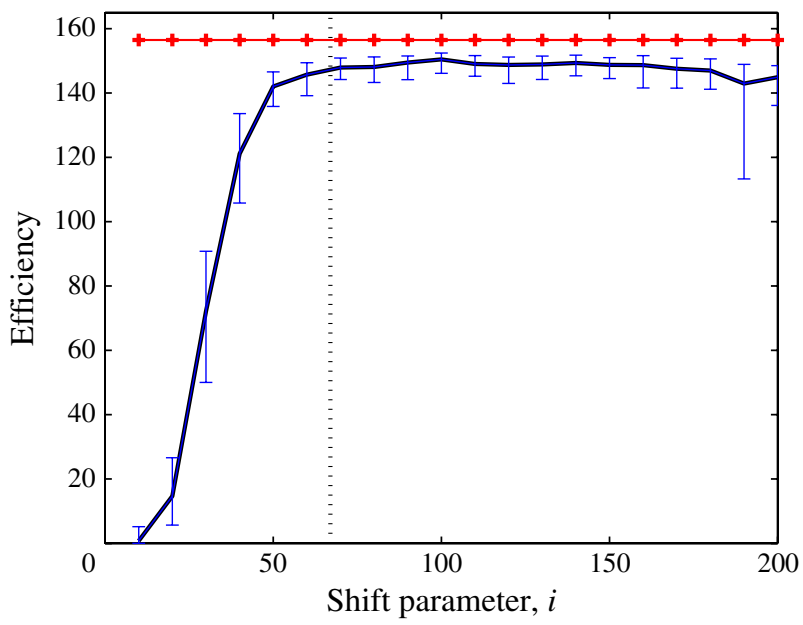

FIGURE 29. (Colour online) Control efficiency of the feed-forward approach as a function of the shift parameter $i$ for the complex Ginzburg-Landau model. Fifty realizations of identification and control were performed; the average (full line with error bars; blue online) and one standard deviation are plotted. These curves are compared to the optimal LQG control designed from the full-order system (full line with plus symbols; red online).

$\begin{array}{lrrr} & \begin{array}{c}\text { White } \\ \text { noise }\end{array} & \begin{array}{c}\text { Random binary } \\ \text { signal }\end{array} & \begin{array}{c}\text { Random triangular } \\ \text { signal }\end{array} \\ \text { Mean efficiency } & 147.1 & 150.5 & 145.4 \\ \text { Standard deviation } & 4.3 & 2.5 & 5.6\end{array}$

TABLE 1. Performance measures of the feed-forward control for the Ginzburg-Landau model for three different input signals.

than the number of time steps needed for the information to travel from the spy sensor to the objective sensor. In other words, the shift $i$ must be larger than $\tau / \Delta t$. In this paper, except when specified otherwise, we chose $i \equiv 1.25 \tau / \Delta t$. In figure 29 , the control efficiency is represented as a function of the shift parameter $i$. The vertical dotted line indicates the parameter $i$ used in our study.

\section{REFERENCES}

BAGHERI, S., BRANDT, L. \& HENNingSON, D. S. $2009 a$ Input-output analysis, model reduction and control of the flat-plate boundary layer. J. Fluid Mech. 620, 263-298.

Bagheri, S., Henningson, D. S., HoepfFner, J. \& Schmid, P. J. $2009 b$ Input-output analysis and control design applied to a linear model of spatially developing flows. Appl. Mech. Rev. 62 (2), 020803.

BARbagallo, A. 2011 Model reduction and closed-loop control of oscillator and noise-amplifier flows. PhD thesis, Ecole Polytechnique, Palaiseau, France.

Barbagallo, A., Dergham, G., SipP, D., Schmid, P. J. \& Robinet, J.-C. 2012 Closed-loop control of unsteadiness over a rounded backward-facing step. J. Fluid Mech. 703, 326-362.

Barkley, D. M., Gomes, M. G. \& Henderson, R. D. 2002 Three-dimensional instability in flow over a backward-facing step. J. Fluid Mech. 473, 167-190. 
Bewley, T. R. 2001 Flow control: new challenges for a new renaissance. Prog. Aerosp. Sci. 37 (1), 21-58.

BEWLEY, T. R. \& LiU, S. 1998 Optimal and robust control and estimation of linear paths to transition. J. Fluid Mech. 365, 305-349.

Blackburn, H. M., Barkley, D. \& Sherwin, S. J. 2008 Convective instability and transient growth in flow over a backward-facing step. J. Fluid Mech. 603, 271-304.

CAlvet, J. P. \& ARKUn, Y. 1988 Feedforward and feedback linearization of nonlinear system and its implementation using internal model control (IMC). Ind. Engng Chem. Res. 27 (10), 1822-1831.

Cattafesta, L., Williams, D., Rowley, C. \& Alvi, F. 2003 Review of active control of flow-induced cavity resonance. AIAA Paper 2003-3567.

Chen, K. K. \& Rowley, C. W. $2011 \mathrm{H}_{2}$ optimal actuator and sensor placement in the linearised complex Ginzburg-Landau system. J. Fluid Mech. 681, 241-260.

Chevalier, M., Heppffner, J., Akervik, E. \& Henningson, D. S. 2007 Linear feedback control and estimation applied to instabilities in spatially developing boundary layers. J. Fluid Mech. 588, 163-187.

Chomaz, J. M. 2005 Global instabilities in spatially developing flows: non-normality and nonlinearity. Annu. Rev. Fluid Mech. 37 (1), 357-392.

Chomaz, J. M., Huerre, P. \& Redekopp, L. G. 1987 Models of hydrodynamic resonances in separated shear flows. In Proceeding of the 6th Symposium on Turbulent Shear Flows, September 7-9, Toulouse, France.

Favoreel, W., De Moor, B., Gevers, M. \& Van Overschee, P. 1998 Model-free subspace-based LQG-design. Tech. Rep., Katholieke Universiteit Leuven.

Friedland, B. 1986 Control System Design, Introduction to State-Space Methods. Dover.

Greenblatt, D. \& Wygnanski, I. J. 2000 The control of flow separation by periodic excitation. Prog. Aerosp. Sci. 36 (7), 487-545.

Henning, L. \& King, R. 2007 Robust multivariable closed-loop control of a turbulent backwardfacing step flow. J. Aircraft 44, 201-208.

Hervé, A., Sipp, D., Schmid, P. J. \& SAmuelides, M. 2010 Reduced order models for control design using system identification. In Proceedings of the 8th European Fluid Mechanics Conference, September.

Hervé, A., Sipp, D., Schmid, P. J. \& SAmuelides, M. 2012 A physics-based approach to flow control using system identification. J. Fluid Mech. 702, 26-58.

Högberg, M., Bewley, T. R. \& Henningson, D. S. 2003 Linear feedback control and estimation of transition in plane channel flow. J. Fluid Mech. 481, 149-175.

Högberg, M. \& Henningson, D. S. 2002 Linear optimal control applied to instabilities in spatially developing boundary layers. J. Fluid Mech. 470, 151-179.

Huerre, P. \& Monkewitz, P. A. 1990 Local and global instabilities in spatially developing flows. Annu. Rev. Fluid Mech. 22, 473-537.

Joshi, S. S., Speyper, J. L. \& Kim, J. 1997 A systems theory approach to the feedback stabilization of infinitesimal and finite-amplitude disturbances in plane Poiseuille flow. J. Fluid Mech. 332, 157-184.

Joslin, R. D. 1998 Aircraft laminar flow control. Annu. Rev. Fluid Mech. 30 (1), 1-29.

JuAng, J.-N., Phan, M., Horta, L. G. \& Longman, R. W. 1991 Identification of observer/Kalman filter Markov parameters - theory and experiments. J. Guid. Control Dyn. 16 (2), 320-329.

KALMAN, R. E. 1960 A new approach to linear filtering and prediction problems. Trans. ASME: J. Basic Engng 82, 35-45.

Kegerise, M., Cabell, O. H. \& Cattafesta, L. N. 2004 Real-time adaptive control of flow-induced cavity tones (invited). AIAA Paper 2004-0572.

Kerstens, W., Pfeiffer, J., Williams, D., King, R. \& Colonius, T. 2011 Closed-loop control of lift for longitudinal gust suppression at low Reynolds numbers. AIAA J. 49 (8).

Kim, J. \& Bewley, T. R. 2007 A linear systems approach to flow control. Annu. Rev. Fluid Mech. 39 (1), 383-417.

Lanzerstorfer, D. \& Kuhlmann, H. C. 2012 Global stability of the two-dimensional flow over a backward-facing step. J. Fluid Mech. 693, 1-27. 
LARIMORE, W. E. 1983 System identification, reduced order filtering and modelling via canonical variate analysis. In Proceedings of the Conference on Decision and Control, IEEE, 22-24 June, pp. 445-451.

LARIMORE, W. E. 1990 Canonical variate analysis in identification, filtering, and adaptive control. In Proceedings of the 29th IEEE Conference on Decision and Control, 5-7 December, Honolulu, Hawaii, vol. 2. pp. 596-604.

DE Larminat, P. 2002 Analyse des Systèmes Linéaires. Hermes Science.

LAUgA, E. \& BEWley, T. R. 2004 Performance of a linear robust control strategy on a nonlinear model of spatially developing flows. J. Fluid Mech. 512, 343-374.

LJUnG, L. 1999 System Identification: Theory for the User, 2nd edn. Prentice-Hall PTR.

Ma, Z., Ahuja, S. \& Rowley, C. 2011 Reduced-order models for control of fluids using the eigensystem realization algorithm. Theor. Comput. Fluid Dyn. 25, 233-247.

MCKelvey, T. 1994 On state-space models in system identification. PhD thesis, Department of Electrical Engineering, Linköping University, Sweden.

Meckl, P. H. \& SeERING, W. P. 1986 Feedforward control techniques to achieve fast settling time in robots. In American Control Conference, IEEE, 18-20 June, 1986, pp. 1913-1918.

Noack, B., Morzynski, M. \& Tadmor, G. 2011 Reduced-Order Modelling for Flow Control. Springer.

QIN, S. J. 2006 An overview of subspace identification. Comput. Chem. Engng 30, 1502-1513.

QIN, S. \& BADGWelL, T. 2003 A survey of industrial model predictive control technology. Control Engng Pract. 11 (7), 733-764.

Rathnasingham, R. \& Breuer, K. S. 2003 Active control of turbulent boundary layers. J. Fluid Mech. 495, 209-233.

Semeraro, O., Bagheri, S., Brandt, L. \& Henningson, D. S. 2011 Feedback control of three-dimensional optimal disturbances using reduced-order models. J. Fluid Mech. 677, 63-102.

Sharma, A. S., Morrison, J. F., McKeon, B. J., Limebeer, D. J. N., Koberg, W. H. \& SHERWIN, S. J. 2011 Relaminarisation of $R e_{\tau}=100$ channel flow with globally stabilising linear feedback control. Phys. Fluids 23 (12), 125105.

Skogestad, S. \& Postlethwaite, I. 1996 Multivariable Feedback Control, Analysis and Design. John Wiley \& Sons.

Thomas, B., Soleimani-Mohseni, M. \& Fahln, P. 2005 Feed-forward in temperature control of buildings. Energy Build. 37 (7), 755-761.

Tian, Y., Song, Q. \& Cattafesta, L. 2006 Adaptive feedback control of flow separation. AIAA Paper 2006-3016.

VAn Overschee, P. \& De Moor, B. 1994 N4SID: subspace algorithms for the identification of combined deterministic-stochastic systems. Automatica 30 (1), 75-93.

VAn Overschee, P. \& De Moor, B. 1995 A unifying theorem for three subspace system identification algorithms. Automatica 31 (12), 1853-1864.

Van Overschee, P. \& De Moor, B. 1996 Subspace Identification for Linear Systems. Kluwer.

Verhaegen, M. \& Deprettere, E. 1991 A fast, recursive MIMO state space model identification algorithm. In Proceedings of the 30th IEEE Conference on Decision and Control, 11-13 December 1991, vol. 2, pp. 1349-1354. IEEE.

ZENG, J. \& DE CALlafon, R. 2003 Feedforward noise cancellation in an airduct using generalized fir filter estimation. In Proceedings of the 42nd IEEE Conference on Decision and Control, 9-12 December 2003, vol. 6, pp. 6392-6397. IEEE.

Zhou, K., Doyle, J. C. \& Glover, K. 1996 Robust and Optimal Control. Prentice-Hall. 